\title{
On sequences of fuzzy sets and fuzzy set-valued mappings
}

\author{
Masamichi Kon ${ }^{1 *}$ and Hiroaki Kuwano ${ }^{2}$
}

"Correspondence:

masakon@cc.hirosaki-u.ac.jp

${ }^{1}$ Graduate School of Science and Technology, Hirosaki University, 3 , Bunkyo, Hirosaki, Aomori 036-8561, Japan

Full list of author information is available at the end of the article

\begin{abstract}
Based on level sets of fuzzy sets, we propose definitions of limits of sequences of fuzzy sets, and limits and derivatives of fuzzy set-valued mappings. Then, their properties are derived. Limits of sequences of fuzzy sets, and limits and derivatives of fuzzy set-valued mappings are fuzzified ones of them for crisp sets, where 'crisp' means 'non-fuzzy'.

MSC: Primary 03E72; secondary 90C70
\end{abstract}

Keywords: sequence of fuzzy sets; fuzzy set-valued mapping; fuzzy set-valued analysis

\section{Introduction and preliminaries}

The usefulness and importance of limits of sequences of crisp sets, and limits (continuity) and derivatives of crisp set-valued mappings have been recognized in many areas, for example, variational analysis, set-valued optimization, stability theory, sensitivity analysis, etc. For details, see, for example, [1-6]. The concept of limits of sequences of crisp sets is interesting and important for itself, and it is necessary to introduce the concepts of limits and derivatives of crisp set-valued mappings. Typical and important applications of them are (i) set-valued optimization and (ii) stability theory and sensitivity analysis for mathematical models. For the case (ii), consider the following system. Some mathematical model outputs the set of optimal values $W^{*}(\mathbf{u}) \subset \mathbb{R}^{\ell}$ and the set of optimal solutions $S^{*}(\mathbf{u}) \subset \mathbb{R}^{n}$ for a given input parameter $\mathbf{u} \in \mathbb{R}^{m}$. Then $W^{*}$ and $S^{*}$ are crisp set-valued mappings. Stability theory deals with the continuity of $W^{*}$ and $S^{*}$. Sensitivity analysis deals with the derivative of $W^{*}$.

In this article, limits of sequences of fuzzy sets, and limits and derivatives of fuzzy setvalued mappings are considered. They are generalizations of them for crisp sets. The aim of this article is to propose those concepts and to investigate their properties systematically.

Some research works deal with limits of sequences of fuzzy numbers or fuzzy sets with bounded supports [7-9], while few research works deal with limits of sequences of fuzzy sets. In addition, some research works deal with limits (continuity) and derivatives of fuzzy number or fuzzy set with bounded support-valued mappings [7,10,11], while few research works deal with limits (continuity) and derivatives of fuzzy set-valued mappings. Furthermore, their approaches need some assumptions that level sets of fuzzy sets are nonempty and compact. Our new approach in this article, however, does not need those assumptions. Limits of sequences of fuzzy sets, and limits and derivatives of fuzzy set-valued mappings

\section{Springer}

○2013 Kon and Kuwano; licensee Springer. This is an Open Access article distributed under the terms of the Creative Commons Attribution License (http://creativecommons.org/licenses/by/2.0), which permits unrestricted use, distribution, and reproduction in any medium, provided the original work is properly cited. 
can be expected to be useful and important for (i) fuzzy set-valued optimization, (ii) stability theory and sensitivity analysis for fuzzy mathematical models, etc. For the case (ii), consider the following system. Some fuzzy mathematical model outputs the fuzzy set of optimal values on $\mathbb{R}^{\ell}, \tilde{W}^{*}(\mathbf{u})$, and the set of optimal solutions $S^{*}(\mathbf{u}) \subset \mathbb{R}^{n}$ for a given input parameter $\mathbf{u} \in \mathbb{R}^{m}$. Then $\tilde{W}^{*}$ is a fuzzy set-valued mapping, and $S^{*}$ is a crisp set-valued mapping. Then we will be able to deal with the continuity of $\tilde{W}^{*}$ and $S^{*}$, and the derivative of $\tilde{W}^{*}$. This means that our proposing approaches give useful tools for stability theory and sensitivity analysis in fuzzy set theory.

We use the following notations.

For $a, b \in \mathbb{R} \cup\{-\infty, \infty\}$, we set $[a, b]=\{x \in \mathbb{R}: a \leq x \leq b\},[a, b[=\{x \in \mathbb{R}: a \leq x<b\}$, ]$a, b]=\{x \in \mathbb{R}: a<x \leq b\}$, and $] a, b[=\{x \in \mathbb{R}: a<x<b\}$.

For $a_{\lambda} \in[0,1], \lambda \in \Lambda$, we define $\bigwedge_{\lambda \in \Lambda} a_{\lambda}=\inf _{\lambda \in \Lambda} a_{\lambda}$ and $\bigvee_{\lambda \in \Lambda} a_{\lambda}=\sup _{\lambda \in \Lambda} a_{\lambda}$, where $\bigwedge_{\lambda \in \Lambda} a_{\lambda}=\inf _{\lambda \in \Lambda} a_{\lambda}=1$ and $\bigvee_{\lambda \in \Lambda} a_{\lambda}=\sup _{\lambda \in \Lambda} a_{\lambda}=0$ if $\Lambda=\emptyset$.

Let $\mathbb{N}$ be the set of all natural numbers, and we set

$$
\begin{aligned}
\mathcal{N}_{\infty} & =\{N \subset \mathbb{N}: \mathbb{N} \backslash N \text { is finite }\} \\
& =\left\{\text { subsequences of } \mathbb{N} \text { containing all } k \text { beyond some } k_{0}\right\}, \\
\mathcal{N}_{\infty}^{\sharp} & =\{N \subset \mathbb{N}: N \text { is infinite }\}=\{\text { all subsequences of } \mathbb{N}\} .
\end{aligned}
$$

A subsequence of a sequence $\left\{x_{k}\right\}_{k \in \mathbb{N}}$ is represented as $\left\{x_{k}\right\}_{k \in N}$ for some $N \in \mathcal{N}_{\infty}^{\sharp}$. We write $\lim _{k}, \lim _{k \rightarrow \infty}$, or $\lim _{k \in \mathbb{N}}$ when $k \rightarrow \infty$ in $\mathbb{N}$, but $\lim _{k \in N}$ or $\lim _{k \rightarrow \infty}$ in the case of the convergence of $\left\{x_{k}\right\}_{k \in N}$ for some $N$ in $\mathcal{N}_{\infty}^{\sharp}$ or $\mathcal{N}_{\infty}$.

For a set $C \subset \mathbb{R}^{n}$, let $\operatorname{cl}(C)$ be the closure of $C$.

Let $\mathcal{C}\left(\mathbb{R}^{n}\right), \mathcal{K}\left(\mathbb{R}^{n}\right)$, and $\mathcal{C K}\left(\mathbb{R}^{n}\right)$ be sets of all closed, convex, and closed convex subsets of $\mathbb{R}^{n}$, respectively.

\subsection{Limits of sequences of sets}

First, we slightly review the definitions of limits of sequences of sets and their properties.

Definition 1.1 (Definition 4.1 in [3]) For a sequence $\left\{C_{k}\right\}_{k \in \mathbb{N}}$ of subsets of $\mathbb{R}^{n}$, its lower limit is defined as the set

$$
\liminf _{k \rightarrow \infty} C_{k}=\left\{\mathbf{x} \in \mathbb{R}^{n}: \exists N \in \mathcal{N}_{\infty}, \exists \mathbf{x}_{k} \in C_{k}(k \in N) \text { with } \mathbf{x}_{k} \underset{N}{\rightarrow} \mathbf{x}\right\}
$$

and its upper limit is defined as the set

$$
\limsup _{k \rightarrow \infty} C_{k}=\left\{\mathbf{x} \in \mathbb{R}^{n}: \exists N \in \mathcal{N}_{\infty}^{\sharp}, \exists \mathbf{x}_{k} \in C_{k}(k \in N) \text { with } \mathbf{x}_{k} \underset{N}{\rightarrow} \mathbf{x}\right\} .
$$

The limit of $\left\{C_{k}\right\}_{k \in \mathbb{N}}$ is said to exist if $\liminf _{k \rightarrow \infty} C_{k}=\lim \sup _{k \rightarrow \infty} C_{k}$, and its limit is defined as the set

$$
\lim _{k \rightarrow \infty} C_{k}=\limsup _{k \rightarrow \infty} C_{k}=\liminf _{k \rightarrow \infty} C_{k}
$$

Example 1.1 For $a, b, c, d \in \mathbb{R}$ with $a<b<c<d$, set $A=[a, c]$ and $B=[b, d]$. For each $k \in \mathbb{N}$, let $C_{k}=A$ if $k$ is odd, and let $C_{k}=B$ if $k$ is even. In this case, we have $\liminf _{k \rightarrow \infty} C_{k}=A \cap B=$ $[b, c]$ and $\limsup _{k \rightarrow \infty} C_{k}=A \cup B=[a, d]$. 
Proposition 1.1 (Exercise 4.2(b) in [3]) For a sequence $\left\{C_{k}\right\}_{k \in \mathbb{N}}$ of subsets of $\mathbb{R}^{n}$, $\liminf _{k} C_{k}=\bigcap_{N \in \mathcal{N}_{\infty}^{\sharp}} \mathrm{cl}\left(\bigcup_{k \in N} C_{k}\right)$ and $\lim \sup _{k} C_{k}=\bigcap_{N \in \mathcal{N}_{\infty}} \operatorname{cl}\left(\bigcup_{k \in N} C_{k}\right)$.

Proposition 1.2 (Exercise 4.3 in [3]) For sequences $\left\{C_{k}\right\}_{k \in \mathbb{N}},\left\{C_{k}^{1}\right\}_{k \in \mathbb{N}},\left\{C_{k}^{2}\right\}_{k \in \mathbb{N}}$ of subsets of $\mathbb{R}^{n}$ and $C \subset \mathbb{R}^{n}$, the following statements hold.

(i) If $C_{k} \nearrow$ (which means that $\left.C_{k} \subset C_{k+1} \subset \cdots\right)$, then $\lim _{k} C_{k}=\operatorname{cl}\left(\bigcup_{k \in \mathbb{N}} C_{k}\right)$.

(ii) If $C_{k} \searrow$ (which means that $\left.C_{k} \supset C_{k+1} \supset \cdots\right)$, then $\lim _{k} C_{k}=\bigcap_{k \in \mathbb{N}} \operatorname{cl}\left(C_{k}\right)$.

(iii) If $C_{k}^{1} \subset C_{k} \subset C_{k}^{2}, k \in \mathbb{N}$ and $C_{k}^{1} \rightarrow C, C_{k}^{2} \rightarrow C$, then $C_{k} \rightarrow C$.

Proposition 1.3 (Proposition 4.4 in [3]) For sequences $\left\{C_{k}\right\}_{k \in \mathbb{N}},\left\{D_{k}\right\}_{k \in \mathbb{N}}$ of subsets of $\mathbb{R}^{n}$ and $C \subset \mathbb{R}^{n}$, the following statements hold.

(i) $\liminf _{k} C_{k}, \limsup \operatorname{su}_{k} C_{k} \in \mathcal{C}\left(\mathbb{R}^{n}\right)$.

(ii) If $\operatorname{cl}\left(C_{k}\right)=\operatorname{cl}\left(D_{k}\right), k \in \mathbb{N}$, then $\liminf _{k} C_{k}=\liminf _{k} D_{k}$ and $\lim \sup _{k} C_{k}=\lim \sup _{k} D_{k}$.

(iii) If $C_{k}=C, k \in \mathbb{N}$, then $\lim _{k} C_{k}=\operatorname{cl}(C)$.

Definition 1.2 (Example 4.13 in [3]) For nonempty closed sets $C, D \subset \mathbb{R}^{n}$, the Hausdorff distance between $C$ and $D$ is defined as

$$
\rho(C, D)=\sup _{\mathbf{x} \in \mathbb{R}^{n}}\left|d_{C}(\mathbf{x})-d_{D}(\mathbf{x})\right|
$$

where $d_{C}(\mathbf{x})=\inf _{\mathbf{y} \in C}\|\mathbf{y}-\mathbf{x}\|, d_{D}(\mathbf{x})=\inf _{\mathbf{y} \in D}\|\mathbf{y}-\mathbf{x}\|$ and $\|\cdot\|$ is the Euclidean norm. A sequence $\left\{C_{k}\right\}_{k \in \mathbb{N}}$ of nonempty closed subsets of $\mathbb{R}^{n}$ is said to converge to a nonempty closed set $C \subset \mathbb{R}^{n}$ with respect to $\rho$ if $\rho\left(C_{k}, C\right) \rightarrow 0$.

Proposition 1.4 (Example 4.13 in [3]) Let $X \subset \mathbb{R}^{n}$ be a bounded set, and let $C_{k} \subset X, k \in \mathbb{N}$ and $C \subset X$ be nonempty closed sets. Then $\left\{C_{k}\right\}_{k \in \mathbb{N}}$ converges to $C$ in the sense of Definition 1.1 if and only if $\left\{C_{k}\right\}_{k \in \mathbb{N}}$ converges to $C$ with respect to $\rho$.

Proposition 1.5 (Proposition 4.15 in [3]) If $\left\{C_{k}\right\}_{k \in \mathbb{N}} \subset \mathcal{K}\left(\mathbb{R}^{n}\right)$, then $\liminf _{k} C_{k} \in \mathcal{K}\left(\mathbb{R}^{n}\right)$.

From Definition 1.1, the following Proposition 1.6 can be obtained.

Proposition 1.6 Let $C_{k} \subset \mathbb{R}^{n}, k \in \mathbb{N}$, and let $C=\lim _{k \rightarrow \infty} C_{k}$. In addition, let $N \in \mathcal{N}_{\infty}^{\sharp}$. Then $C=\lim _{k \rightarrow \infty} C_{k}$.

\subsection{Limits of set-valued mappings}

Next, we give the definitions of limits of set-valued mappings and their properties.

A mapping $F$ such that $F(\mathbf{x}) \subset \mathbb{R}^{m}$ for each $\mathbf{x} \in \mathbb{R}^{n}$ is called a set-valued mapping from $\mathbb{R}^{n}$ to $\mathbb{R}^{m}$, and it is denoted by $F: \mathbb{R}^{n} \rightsquigarrow \mathbb{R}^{m}$. The set-valued mapping $F$ is said to be closedvalued, convex-valued, or closed convex-valued if $F(\mathbf{x}) \in \mathcal{C}\left(\mathbb{R}^{m}\right), F(\mathbf{x}) \in \mathcal{K}\left(\mathbb{R}^{m}\right)$, or $F(\mathbf{x}) \in$ $\mathcal{C K}\left(\mathbb{R}^{m}\right)$ for any $\mathbf{x} \in \mathbb{R}^{n}$, respectively.

Definition 1.3 (See p.152 in [3]) Let $F: \mathbb{R}^{n} \rightsquigarrow \mathbb{R}^{m}$, and let $\overline{\mathbf{x}} \in \mathbb{R}^{n}$. The lower limit of $F$ when $\mathbf{x} \rightarrow \overline{\mathbf{x}}$ is defined as the set

$$
\liminf _{\mathbf{x} \rightarrow \overline{\mathbf{x}}} F(\mathbf{x})=\bigcap_{\mathbf{x}_{k} \rightarrow \overline{\mathbf{x}}} \liminf _{k \rightarrow \infty} F\left(\mathbf{x}_{k}\right)
$$


and the upper limit of $F$ when $\mathbf{x} \rightarrow \overline{\mathbf{x}}$ is defined as the set

$$
\limsup _{\mathbf{x} \rightarrow \overline{\mathbf{x}}} F(\mathbf{x})=\bigcup_{\mathbf{x}_{k} \rightarrow \overline{\mathbf{x}}} \limsup _{k \rightarrow \infty} F\left(\mathbf{x}_{k}\right)
$$

where $\bigcap_{\mathbf{x}_{k} \rightarrow \overline{\mathbf{x}}}$ and $\bigcup_{\mathbf{x}_{k} \rightarrow \overline{\mathbf{x}}}$ mean the intersection and the union with respect to any sequence $\left\{\mathbf{x}_{k}\right\}_{k \in \mathbb{N}} \subset \mathbb{R}^{n}$ such that $\mathbf{x}_{k} \rightarrow \overline{\mathbf{x}}$, respectively. The limit of $F$ when $\mathbf{x} \rightarrow \overline{\mathbf{x}}$ is said to

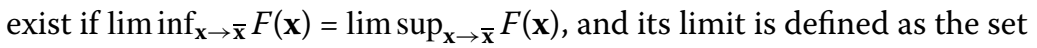

$$
\lim _{\mathbf{x} \rightarrow \overline{\mathbf{x}}} F(\mathbf{x})=\liminf _{\mathbf{x} \rightarrow \overline{\mathbf{x}}} F(\mathbf{x})=\limsup _{\mathbf{x} \rightarrow \overline{\mathbf{x}}} F(\mathbf{x})
$$

Example 1.2 For $a, b, c, d \in \mathbb{R}$ with $a<b<c<d$, set $A=[b, c]$ and $B=[a, d]$. Let $F, G: \mathbb{R} \rightsquigarrow$ $\mathbb{R}$ be set-valued mappings defined as

$$
F(x)=\left\{\begin{array}{ll}
A & \text { if } x \leq 0, \\
B & \text { if } x>0,
\end{array} \quad G(x)= \begin{cases}A & \text { if } x<0 \\
B & \text { if } x \geq 0\end{cases}\right.
$$

for each $x \in \mathbb{R}$. In this case, we have $\liminf _{x \rightarrow 0} F(x)=\liminf _{x \rightarrow 0} G(x)=A=[b, c]$ and $\lim \sup _{x \rightarrow 0} F(x)=\lim \sup _{x \rightarrow 0} G(x)=B=[a, d]$.

Definition 1.4 (Definition 5.4 in [3]) Let $F: \mathbb{R}^{n} \rightsquigarrow \mathbb{R}^{m}$, and let $\overline{\mathbf{x}} \in \mathbb{R}^{n}$. The set-valued mapping $F$ is said to be lower semicontinuous at $\overline{\mathbf{x}}$ if

$$
\liminf _{\mathbf{x} \rightarrow \overline{\mathbf{x}}} F(\mathbf{x}) \supset F(\overline{\mathbf{x}}),
$$

and $F$ is said to be upper semicontinuous at $\overline{\mathbf{x}}$ if

$$
\limsup _{\mathbf{x} \rightarrow \overline{\mathbf{x}}} F(\mathbf{x}) \subset F(\overline{\mathbf{x}})
$$

The set-valued mapping $F$ is said to be continuous at $\overline{\mathbf{x}}$ if $F$ is both lower and upper semicontinuous at $\overline{\mathbf{x}}$, that is,

$$
\lim _{\mathbf{x} \rightarrow \overline{\mathbf{x}}} F(\mathbf{x})=F(\overline{\mathbf{x}}) .
$$

Example 1.3 Consider the set-valued mappings $F$ and $G$ defined in Example 1.2. Since $\liminf _{x \rightarrow 0} F(x)=[b, c] \supset[b, c]=F(0)$ and $\limsup _{x \rightarrow 0} F(x)=[a, d] \not \subset[b, c]=F(0), F$ is lower semicontinuous at 0 but not upper semicontinuous at 0 . Since $\lim _{\sup _{x \rightarrow 0}} G(x)=[a, d] \subset$ $[a, d]=G(0)$ and $\liminf _{x \rightarrow 0} G(x)=[b, c] \not \supset[a, d]=G(0), G$ is upper semicontinuous at 0 but not lower semicontinuous at 0 .

From Definition 1.3, the following Proposition 1.7 can be obtained.

Proposition 1.7 Let $F: \mathbb{R}^{n} \rightsquigarrow \mathbb{R}^{m}$, and let $\overline{\mathbf{x}} \in \mathbb{R}^{n}$. Then $\lim _{\mathbf{x} \rightarrow \overline{\mathbf{x}}} F(\mathbf{x})=F(\overline{\mathbf{x}})$ if and only if $\lim _{k} F\left(\mathbf{x}_{k}\right)=F(\overline{\mathbf{x}})$ for any sequence $\left\{\mathbf{x}_{k}\right\}_{k \in \mathbb{N}} \subset \mathbb{R}^{n}$ such that $\mathbf{x}_{k} \rightarrow \overline{\mathbf{x}}$. 


\subsection{Derivatives of set-valued mappings}

In this subsection, we give the definition of derivatives of set-valued mappings and their properties.

A set $C \subset \mathbb{R}^{n}$ is called a cone if $\mathbf{0} \in C$ and $\lambda \mathbf{x} \in C$ for any $\mathbf{x} \in C$ and any $\lambda \geq 0$.

Definition 1.5 (Definition 3.4 in [2]) Let $S \subset \mathbb{R}^{n}$, and let $\mathbf{x}_{0} \in S$. A vector $\mathbf{d} \in \mathbb{R}^{n}$ is called a tangent vector of $S$ at $\mathbf{x}_{0}$ if there exists a sequence $\left\{\mathbf{x}_{k}\right\}_{k \in \mathbb{N}} \subset S$, which converges to $\mathbf{x}_{0}$, and a sequence $\left\{t_{k}\right\}_{k \in \mathbb{N}} \subset \mathbb{R}$ of positive real numbers such that

$$
\lim _{k \rightarrow \infty} t_{k}\left(\mathbf{x}_{k}-\mathbf{x}_{0}\right)=\mathbf{d}
$$

The set of all tangent vectors of $S$ at $\mathbf{x}_{0}$ is called the tangent cone of $S$ at $\mathbf{x}_{0}$, and it is denoted by $T\left(S ; \mathbf{x}_{0}\right)$.

A tangent cone in Definition 1.5 is also called a contingent cone. For details of tangent cones, see, for example, $[1,12]$.

Example 1.4 Let $\alpha \in] 0,1]$, and let $S=\left\{(x, y) \in \mathbb{R}^{2}: \min \left\{\alpha\left(x^{3}-x\right),(2-\alpha)\left(x^{3}-x\right)\right\} \leq\right.$ $\left.y \leq \max \left\{\alpha\left(x^{3}-x\right),(2-\alpha)\left(x^{3}-x\right)\right\}\right\}$. In addition, let $\mathbf{x}_{0}=(0,0) \in S$. In this case, we have $T\left(S ; \mathbf{x}_{0}\right)=\left\{(x, y) \in \mathbb{R}^{2}: \min \{-\alpha x,-(2-\alpha) x\} \leq y \leq \max \{-\alpha x,-(2-\alpha) x\}\right\}$.

Proposition 1.8 (Theorem 3.7 in [2]) Let $S \subset \mathbb{R}^{n}$, and let $\mathbf{x}_{0} \in S$. Then $T\left(S ; \mathbf{x}_{0}\right)$ is a closed cone. Furthermore, if $S$ is convex, then $T\left(S ; \mathbf{x}_{0}\right)$ is also convex.

Proposition 1.9 (Theorem 3.8 in [2]) Let $S, Q \subset \mathbb{R}^{n}$ with $S \subset Q$, and let $\mathbf{x}_{0} \in S$. Then $T\left(S ; \mathbf{x}_{0}\right) \subset T\left(Q ; \mathbf{x}_{0}\right)$.

For a set-valued mapping $F: \mathbb{R}^{n} \rightsquigarrow \mathbb{R}^{m}$, the set

$$
\operatorname{Graph}(F)=\left\{(\mathbf{x}, \mathbf{y}) \in \mathbb{R}^{n} \times \mathbb{R}^{m}: \mathbf{y} \in F(\mathbf{x})\right\}
$$

is called the graph of $F$.

Definition 1.6 (Definition 4.8 in [2], Definition 5.1.1 in [1]) Let $F: \mathbb{R}^{n} \rightsquigarrow \mathbb{R}^{m}$, and let $\left(\mathbf{x}_{0}, \mathbf{y}_{0}\right) \in \operatorname{Graph}(F)$. Then the set-valued mapping $D F\left(\mathbf{x}_{0}, \mathbf{y}_{0}\right): \mathbb{R}^{n} \rightsquigarrow \mathbb{R}^{m}$ defined as

$$
D F\left(\mathbf{x}_{0}, \mathbf{y}_{0}\right)(\mathbf{u})=\left\{\mathbf{v} \in \mathbb{R}^{m}:(\mathbf{u}, \mathbf{v}) \in T\left(\operatorname{Graph}(F) ;\left(\mathbf{x}_{0}, \mathbf{y}_{0}\right)\right)\right\}
$$

for each $\mathbf{u} \in \mathbb{R}^{n}$ is called the contingent derivative of $F$ at $\left(\mathbf{x}_{0}, \mathbf{y}_{0}\right)$.

From Definition 1.6, it can be seen that

$$
\operatorname{Graph}\left(D F\left(\mathbf{x}_{0}, \mathbf{y}_{0}\right)\right)=T\left(\operatorname{Graph}(F) ;\left(\mathbf{x}_{0}, \mathbf{y}_{0}\right)\right)
$$

Example 1.5 Let $\alpha \in] 0,1]$, and let $F: \mathbb{R} \rightsquigarrow \mathbb{R}$ be a set-valued mapping defined as $F(x)=$ $\left[\min \left\{\alpha\left(x^{3}-x\right),(2-\alpha)\left(x^{3}-x\right)\right\}, \max \left\{\alpha\left(x^{3}-x\right),(2-\alpha)\left(x^{3}-x\right)\right\}\right]$. Then $(0,0) \in \operatorname{Graph}(F)$. From Example 1.4, we have $D F(0,0)(u)=[\min \{-\alpha u,-(2-\alpha) u\}, \max \{-\alpha u,-(2-\alpha) u\}]$ for each $u \in \mathbb{R}$. 
From Definition 1.6 and Proposition 1.8, the following Proposition 1.10 can be obtained.

Proposition 1.10 Let $F: \mathbb{R}^{n} \rightsquigarrow \mathbb{R}^{m}$, and let $\left(\mathbf{x}_{0}, \mathbf{y}_{0}\right) \in \operatorname{Graph}(F)$. Then the following statements hold.

(i) $D F\left(\mathbf{x}_{0}, \mathbf{y}_{0}\right)$ is closed-valued.

(ii) If $\operatorname{Graph}(F) \in \mathcal{K}\left(\mathbb{R}^{n} \times \mathbb{R}^{m}\right)$, then $D F\left(\mathbf{x}_{0}, \mathbf{y}_{0}\right)$ is closed convex-valued.

\subsection{Fuzzy sets}

We investigate properties of level sets of fuzzy sets, which are necessary to consider limits of sequences of fuzzy sets, and limits and derivatives of fuzzy set-valued mappings.

We identify each fuzzy set $\tilde{s}$ on $\mathbb{R}^{n}$ with its membership function, and $\tilde{s}$ is interpreted as $\tilde{s}: \mathbb{R}^{n} \rightarrow[0,1]$. Let $\mathcal{F}\left(\mathbb{R}^{n}\right)$ be the set of all fuzzy sets on $\mathbb{R}^{n}$.

For $\tilde{s} \in \mathcal{F}\left(\mathbb{R}^{n}\right)$ and $\alpha \in[0,1]$, the set

$$
[\tilde{s}]_{\alpha}=\left\{\mathbf{x} \in \mathbb{R}^{n}: \tilde{s}(\mathbf{x}) \geq \alpha\right\}
$$

is called the $\alpha$-level set of $\tilde{s}$.

For a crisp set $S \subset \mathbb{R}^{n}$, the function $c_{S}: \mathbb{R}^{n} \rightarrow\{0,1\}$ defined as

$$
c_{S}(\mathbf{x})= \begin{cases}1 & \text { if } \mathbf{x} \in S, \\ 0 & \text { if } \mathbf{x} \notin S\end{cases}
$$

for each $\mathbf{x} \in \mathbb{R}^{n}$ is called the indicator function of $S$. Whenever we consider $c_{S}$ as a fuzzy set, $c_{S}: \mathbb{R}^{n} \rightarrow\{0,1\}$ is interpreted as $c_{S}: \mathbb{R}^{n} \rightarrow[0,1]$.

It is known as the decomposition theorem that $\tilde{s} \in \mathcal{F}\left(\mathbb{R}^{n}\right)$ can be represented as

$$
\tilde{s}=\sup _{\alpha \in] 0,1]} \alpha c_{[\tilde{s}]_{\alpha}}
$$

(see, for example, [13]).

A fuzzy set $\tilde{s} \in \mathcal{F}\left(\mathbb{R}^{n}\right)$ is said to be closed if $\tilde{s}$ is upper semicontinuous. A fuzzy set $\tilde{s} \in$ $\mathcal{F}\left(\mathbb{R}^{n}\right)$ is closed if and only if $\left.\left.[\tilde{s}]_{\alpha} \in \mathcal{C}\left(\mathbb{R}^{n}\right), \alpha \in\right] 0,1\right]$.

A fuzzy set $\tilde{s} \in \mathcal{F}\left(\mathbb{R}^{n}\right)$ is said to be convex if

$$
\tilde{s}(\lambda \mathbf{x}+(1-\lambda) \mathbf{y}) \geq \tilde{s}(\mathbf{x}) \wedge \tilde{s}(\mathbf{y})
$$

for any $\mathbf{x}, \mathbf{y} \in \mathbb{R}^{n}$ and any $\lambda \in[0,1]$. Namely, $\tilde{s} \in \mathcal{F}\left(\mathbb{R}^{n}\right)$ is said to be convex if $\tilde{s}$ is a quasiconcave function, and $\tilde{s}$ is convex if and only if $\left.\left.[\tilde{s}]_{\alpha} \in \mathcal{K}\left(\mathbb{R}^{n}\right), \alpha \in\right] 0,1\right]$.

Let $\mathcal{C F}\left(\mathbb{R}^{n}\right), \mathcal{K} \mathcal{F}\left(\mathbb{R}^{n}\right)$, and $\mathcal{C K} \mathcal{F}\left(\mathbb{R}^{n}\right)$ be sets of all closed, convex, and closed convex fuzzy sets on $\mathbb{R}^{n}$, respectively.

A fuzzy set $\tilde{s} \in \mathcal{F}\left(\mathbb{R}^{n}\right)$ is called a fuzzy cone if $\left.\left.[\tilde{s}]_{\alpha} \subset \mathbb{R}^{n}, \alpha \in\right] 0,1\right]$ are cones.

For $\tilde{s} \in \mathcal{F}\left(\mathbb{R}^{n}\right)$, the set

$$
\operatorname{hypo}(\tilde{s})=\left\{(\mathbf{x}, \alpha) \in \mathbb{R}^{n} \times[0,1]: \alpha \leq \tilde{s}(\mathbf{x})\right\}
$$

is called the fuzzy hypograph of $\tilde{s}$. 
In order to investigate relationships between fuzzy sets and their level sets, we set

$$
\left.\left.\left.\left.\mathcal{S}\left(\mathbb{R}^{n}\right)=\left\{\left\{S_{\alpha}\right\}_{\alpha \in] 0,1]}: S_{\alpha} \subset \mathbb{R}^{n}, \alpha \in\right] 0,1\right] \text {, and } S_{\beta} \supset S_{\gamma} \text { for } \beta, \gamma \in\right] 0,1\right] \text { with } \beta<\gamma\right\} \text {, }
$$

and define $M: \mathcal{S}\left(\mathbb{R}^{n}\right) \rightarrow \mathcal{F}\left(\mathbb{R}^{n}\right)$ as

$$
M\left(\left\{S_{\alpha}\right\}_{\alpha \in] 0,1]}\right)=\sup _{\alpha \in] 0,1]} \alpha c_{S_{\alpha}}
$$

for each $\left\{S_{\alpha}\right\}_{\alpha \in] 0,1]} \in \mathcal{S}\left(\mathbb{R}^{n}\right)$. For $\left\{S_{\alpha}\right\}_{\alpha \in] 0,1]} \in \mathcal{S}\left(\mathbb{R}^{n}\right)$ and $\mathbf{x} \in \mathbb{R}^{n}$, it can be seen that

$$
\left.\left.M\left(\left\{S_{\alpha}\right\}_{\alpha \in] 0,1]}\right)(\mathbf{x})=\sup _{\alpha \in] 0,1]} \alpha c_{S_{\alpha}}(\mathbf{x})=\sup \{\alpha \in] 0,1\right]: \mathbf{x} \in S_{\alpha}\right\}
$$

where $\sup \emptyset=0$. By using $M$, the decomposition theorem can be represented as

$$
\tilde{s}=M\left(\left\{[\tilde{s}]_{\alpha}\right\}_{\alpha \in] 0,1]}\right)
$$

for $\tilde{s} \in \mathcal{F}\left(\mathbb{R}^{n}\right)$.

Proposition 1.11 (Proposition 3 in [14]) Let $\left\{S_{\alpha}\right\}_{\alpha \in] 0,1]},\left\{T_{\alpha}\right\}_{\alpha \in] 0,1]} \in \mathcal{S}\left(\mathbb{R}^{n}\right)$. If $S_{\alpha} \subset T_{\alpha}$, $\alpha \in] 0,1]$, then $M\left(\left\{S_{\alpha}\right\}_{\alpha \in] 0,1]}\right) \leq M\left(\left\{T_{\alpha}\right\}_{\alpha \in] 0,1]}\right)$.

Proposition 1.12 (Proposition 4 in [14]) Let $\left\{S_{\alpha}\right\}_{\alpha \in] 0,1]} \in \mathcal{S}\left(\mathbb{R}^{n}\right)$, and let $\tilde{s}=M\left(\left\{S_{\alpha}\right\}_{\alpha \in] 0,1]}\right)$. Then $[\tilde{s}]_{\alpha}=\bigcap_{\beta \in] 0, \alpha[} S_{\beta}$ for $\left.\left.\alpha \in\right] 0,1\right]$.

Definition 1.7 For $\tilde{s} \in \mathcal{F}\left(\mathbb{R}^{n}\right)$, the fuzzy set

$$
\operatorname{cl}(\tilde{s})=M\left(\left\{\operatorname{cl}\left([\tilde{s}]_{\alpha}\right)\right\}_{\alpha \in] 0,1]}\right)
$$

is called the closure of $\tilde{s}$.

For a crisp set $S \subset \mathbb{R}^{n}$, it can be seen that $\operatorname{cl}\left(c_{S}\right)=c_{\mathrm{cl}(S)}$. Thus, the closure for fuzzy sets is a generalization of the crisp one.

Proposition 1.13 (See pp.13-14 in [3]) Let $\tilde{s} \in \mathcal{F}\left(\mathbb{R}^{n}\right)$, and let $\tilde{u} \in \mathcal{C F}\left(\mathbb{R}^{n}\right)$ be the smallest closed fuzzy set among closed fuzzy sets $\tilde{t} \in \mathcal{C F}\left(\mathbb{R}^{n}\right)$ such that $\tilde{s} \leq \tilde{t}$. Then hypo $(\tilde{u})=$ $\operatorname{cl}(\operatorname{hypo}(\tilde{s}))$.

Proposition 1.14 Let $\tilde{s} \in \mathcal{F}\left(\mathbb{R}^{n}\right)$. Then the following statements hold.

(i) $[\operatorname{cl}(\tilde{s})]_{\alpha}=\bigcap_{\beta \in] 0, \alpha[} \operatorname{cl}\left([\tilde{s}]_{\beta}\right)$ for $\left.\left.\alpha \in\right] 0,1\right]$.

(ii) $\operatorname{cl}(\tilde{s}) \in \mathcal{C F}\left(\mathbb{R}^{n}\right)$.

(iii) $\operatorname{cl}(\tilde{s})$ is the smallest closed fuzzy set among closed fuzzy sets $\tilde{t} \in \mathcal{C F}\left(\mathbb{R}^{n}\right)$ such that $\tilde{s} \leq \tilde{t}$.

Proof (i) follows from Proposition 1.12. (ii) follows from (i). In order to show (iii), let $\tilde{u} \in$ $\mathcal{C F}\left(\mathbb{R}^{n}\right)$ be the smallest closed fuzzy set among closed fuzzy sets $\tilde{t} \in \mathcal{C} \mathcal{F}\left(\mathbb{R}^{n}\right)$ such that $\tilde{s} \leq \tilde{t}$, and we show that $\tilde{u}=\operatorname{cl}(\tilde{s})$. It follows that $\operatorname{cl}(\tilde{s}) \in \mathcal{C} \mathcal{F}\left(\mathbb{R}^{n}\right)$ from (ii), and that $\tilde{s} \leq \operatorname{cl}(\tilde{s})$ 
from the decomposition theorem and Proposition 1.11. Thus, we have $\tilde{u} \leq \operatorname{cl}(\tilde{s})$. Assume that there exists $\mathbf{x}_{0} \in \mathbb{R}^{n}$ such that $\tilde{u}\left(\mathbf{x}_{0}\right)<\operatorname{cl}(\tilde{s})\left(\mathbf{x}_{0}\right)$. We set $\alpha=\operatorname{cl}(\tilde{s})\left(\mathbf{x}_{0}\right)$ and $\beta=\tilde{u}\left(\mathbf{x}_{0}\right)$, and fix any $\gamma \in] \beta, \alpha\left[\right.$. Since $\left.\left.\alpha=\operatorname{cl}(\tilde{s})\left(\mathbf{x}_{0}\right)=\sup \{\eta \in] 0,1\right]: \mathbf{x}_{0} \in \operatorname{cl}\left([\tilde{s}]_{\eta}\right)\right\}$, it follows that

$$
\mathbf{x}_{0} \in \operatorname{cl}\left([\tilde{s}]_{\gamma}\right)
$$

Since $\beta<\gamma$, it follows that

$$
\left(\mathbf{x}_{0}, \gamma\right) \notin \operatorname{hypo}(\tilde{u})=\operatorname{cl}(\operatorname{hypo}(\tilde{s}))
$$

from Proposition 1.13. From (1.1), it follows that there exists $\left\{\mathbf{x}_{k}\right\}_{k \in \mathbb{N}} \subset[\tilde{s}]_{\gamma}$ such that $\mathbf{x}_{k} \rightarrow \mathbf{x}_{0}$. Since $\tilde{s}\left(\mathbf{x}_{k}\right) \geq \gamma, k \in \mathbb{N}$, we have $\left(\mathbf{x}_{k}, \gamma\right) \in \operatorname{hypo}(\tilde{s}), k \in \mathbb{N}$ and $\left(\mathbf{x}_{k}, \gamma\right) \rightarrow\left(\mathbf{x}_{0}, \gamma\right) \in$ cl(hypo $(\tilde{s}))$, which contradicts (1.2).

The following Example 1.6 shows that $[\operatorname{cl}(\tilde{s})]_{\alpha}=\operatorname{cl}\left([\tilde{s}]_{\alpha}\right)$ does not hold in general.

Example 1.6 Let $\tilde{s} \in \mathcal{F}(\mathbb{R})$ be a fuzzy set defined as

$$
\tilde{s}(x)= \begin{cases}\max \{-|x|+1,0\} & \text { if } x \neq 0 \\ 0 & \text { if } x=0\end{cases}
$$

for each $x \in \mathbb{R}$. In this case, we have $\operatorname{cl}(\tilde{s})(x)=\max \{-|x|+1,0\}$ for each $x \in \mathbb{R}$, and $\operatorname{cl}\left([\tilde{s}]_{1}\right)=$ $\emptyset \neq\{0\}=[\operatorname{cl}(\tilde{s})]_{1}$.

From Propositions 1.11, 1.12, and 1.14, the following Proposition 1.15 can be obtained.

Proposition 1.15 Let $\left\{S_{\alpha}\right\}_{\alpha \in] 0,1]} \in \mathcal{S}\left(\mathbb{R}^{n}\right)$, and let $\tilde{s}=M\left(\left\{S_{\alpha}\right\}_{\alpha \in] 0,1]}\right)$. Then $\operatorname{cl}(\tilde{s})=$ $M\left(\left\{\operatorname{cl}\left(S_{\alpha}\right)\right\}_{\alpha \in] 0,1]}\right)$.

Proposition 1.16 Let $\left\{S_{\alpha}^{(\lambda)}\right\}_{\alpha \in] 0,1]} \in \mathcal{S}\left(\mathbb{R}^{n}\right), \lambda \in \Lambda$, and let $\tilde{s}_{\lambda}=M\left(\left\{S_{\alpha}^{(\lambda)}\right\}_{\alpha \in] 0,1]}\right), \lambda \in \Lambda$. In addition, let $L_{\alpha}=\bigcap_{\lambda \in \Lambda} S_{\alpha}^{(\lambda)}, U_{\alpha}=\bigcup_{\lambda \in \Lambda} S_{\alpha}^{(\lambda)}$ for each $\left.\left.\alpha \in\right] 0,1\right]$, where $L_{\alpha}=\bigcap_{\lambda \in \Lambda} S_{\alpha}^{(\lambda)}=$ $\mathbb{R}^{n}, U_{\alpha}=\bigcup_{\lambda \in \Lambda} S_{\alpha}^{(\lambda)}=\emptyset$ for each $\left.\left.\alpha \in\right] 0,1\right]$ if $\Lambda=\emptyset$. Then $M\left(\left\{L_{\alpha}\right\}_{\alpha \in] 0,1]}\right)=\bigwedge_{\lambda \in \Lambda} \tilde{s}_{\lambda}$ and $M\left(\left\{U_{\alpha}\right\}_{\alpha \in] 0,1]}\right)=\bigvee_{\lambda \in \Lambda} \tilde{s}_{\lambda}$.

Proof Fix any $\mathbf{x} \in \mathbb{R}^{n}$

First, assume that $\Lambda=\emptyset$. Then it follows that $L_{\alpha}=\mathbb{R}^{n}, U_{\alpha}=\emptyset$ for each $\left.\left.\alpha \in\right] 0,1\right]$, and we have $M\left(\left\{L_{\alpha}\right\}_{\alpha \in] 0,1]}\right)(\mathbf{x})=1=\bigwedge_{\lambda \in \Lambda} \tilde{s}_{\lambda}(\mathbf{x})$ and $M\left(\left\{U_{\alpha}\right\}_{\alpha \in] 0,1]}\right)(\mathbf{x})=0=\bigvee_{\lambda \in \Lambda} \tilde{s}_{\lambda}(\mathbf{x})$.

Next, assume that $\Lambda \neq \emptyset$. We set $\left.\left.\beta=M\left(\left\{L_{\alpha}\right\}_{\alpha \in] 0,1]}\right)(\mathbf{x})=\sup \{\alpha \in] 0,1\right]: \mathbf{x} \in L_{\alpha}\right\}$ and $\gamma=$ $\left.\left.M\left(\left\{U_{\alpha}\right\}_{\alpha \in] 0,1]}\right)(\mathbf{x})=\sup \{\alpha \in] 0,1\right]: \mathbf{x} \in U_{\alpha}\right\}$.

From the definition of $\beta$, it follows that $\left.\mathbf{x} \in L_{\alpha}, \alpha \in\right] 0, \beta\left[\right.$ and $\left.\left.\mathbf{x} \notin L_{\alpha}, \alpha \in\right] \beta, 1\right]$. For any $\lambda \in \Lambda$ and any $\alpha \in] 0, \beta$ [, it follows that $\tilde{s}_{\lambda}(\mathbf{x}) \geq \alpha$ since $\mathbf{x} \in S_{\alpha}^{(\lambda)}$. Thus, $\tilde{s}_{\lambda}(\mathbf{x}) \geq \beta$ for any $\lambda \in \Lambda$. For each $\alpha \in] \beta, 1]$, since $\mathbf{x} \notin L_{\alpha}$, there exists $\lambda_{\alpha} \in \Lambda$ such that $\mathbf{x} \notin S_{\alpha}^{\left(\lambda_{\alpha}\right)}$, and it follows that $\tilde{s}_{\lambda_{\alpha}}(\mathbf{x}) \leq \alpha$. Thus, for any $\varepsilon>0$, there exists $\lambda_{0} \in \Lambda$ such that $\tilde{s}_{\lambda_{0}}(\mathbf{x})<\beta+\varepsilon$. Therefore, we have $\beta=\bigwedge_{\lambda \in \Lambda} \tilde{s}_{\lambda}(\mathbf{x})$.

By the same way, we have $\gamma=\bigvee_{\lambda \in \Lambda} \tilde{s}_{\lambda}(\mathbf{x})$.

Proposition 1.17 Let $\left\{S_{\alpha}\right\}_{\alpha \in] 0,1]},\left\{T_{\alpha}\right\}_{\alpha \in] 0,1]} \in \mathcal{S}\left(\mathbb{R}^{n}\right)$. If $S_{\alpha}=T_{\alpha}$ for any $\left.\left.\alpha \in\right] 0,1\right]$ except for at most countable many $\alpha$, then $M\left(\left\{S_{\alpha}\right\}_{\alpha \in] 0,1]}\right)=M\left(\left\{T_{\alpha}\right\}_{\alpha \in] 0,1]}\right)$. 
Proof Assume that there exists $\mathbf{x}_{0} \in \mathbb{R}^{n}$ such that $M\left(\left\{S_{\alpha}\right\}_{\alpha \in] 0,1]}\right)\left(\mathbf{x}_{0}\right) \neq M\left(\left\{T_{\alpha}\right\}_{\alpha \in] 0,1]}\right)\left(\mathbf{x}_{0}\right)$. Without loss of generality, assume that $M\left(\left\{S_{\alpha}\right\}_{\alpha \in] 0,1]}\right)\left(\mathbf{x}_{0}\right)<M\left(\left\{T_{\alpha}\right\}_{\alpha \in] 0,1]}\right)\left(\mathbf{x}_{0}\right)$. We set $\beta=$ $\left.\left.M\left(\left\{S_{\alpha}\right\}_{\alpha \in] 0,1]}\right)\left(\mathbf{x}_{0}\right)=\sup \{\alpha \in] 0,1\right]: \mathbf{x}_{0} \in S_{\alpha}\right\}$ and $\left.\gamma=M\left(\left\{T_{\alpha}\right\}_{\alpha \in] 0,1]}\right)\left(\mathbf{x}_{0}\right)=\sup \{\alpha \in] 0,1\right]$ : $\left.\mathbf{x}_{0} \in T_{\alpha}\right\}$. Since

$$
\left.\beta=\sup \{\alpha \in] 0,1]: \mathbf{x}_{0} \in S_{\alpha}\right\} \quad \Leftrightarrow \quad \begin{cases}\mathbf{x}_{0} \in S_{\alpha}, & \alpha \in] 0, \beta[ \\ \mathbf{x}_{0} \notin S_{\alpha}, & \alpha \in] \beta, 1]\end{cases}
$$

and

$$
\left.\gamma=\sup \{\alpha \in] 0,1]: \mathbf{x}_{0} \in T_{\alpha}\right\} \quad \Leftrightarrow \quad \begin{cases}\mathbf{x}_{0} \in T_{\alpha}, & \alpha \in] 0, \gamma[ \\ \mathbf{x}_{0} \notin T_{\alpha}, & \alpha \in] \gamma, 1]\end{cases}
$$

we have $\left.\mathbf{x}_{0} \notin S_{\alpha}, \mathbf{x}_{0} \in T_{\alpha}, \alpha \in\right] \beta, \gamma\left[\right.$ and $\left.S_{\alpha} \neq T_{\alpha}, \alpha \in\right] \beta, \gamma[$. Therefore, it is not true that $S_{\alpha}=T_{\alpha}$ for any $\left.\left.\alpha \in\right] 0,1\right]$ except for at most countable many $\alpha$.

\section{Limits of sequences of fuzzy sets}

In this section, we propose the definitions of limits of sequences of fuzzy sets based on their level sets, and investigate their properties.

The following Definition 2.1 is a fuzzified one of Definition 1.1.

Definition 2.1 Let $\left\{\tilde{s}_{k}\right\}_{k \in \mathbb{N}} \subset \mathcal{F}\left(\mathbb{R}^{n}\right)$, and let

$$
L_{\alpha}=\liminf _{k \rightarrow \infty}\left[\tilde{s}_{k}\right]_{\alpha}, \quad U_{\alpha}=\limsup _{k \rightarrow \infty}\left[\tilde{s}_{k}\right]_{\alpha}
$$

for each $\alpha \in] 0,1]$. The lower limit of $\left\{\tilde{s}_{k}\right\}_{k \in \mathbb{N}}$ is defined as the fuzzy set

$$
\liminf _{k \rightarrow \infty} \tilde{s}_{k}=M\left(\left\{L_{\alpha}\right\}_{\alpha \in] 0,1]}\right)
$$

and the upper limit of $\left\{\tilde{s}_{k}\right\}_{k \in \mathbb{N}}$ is defined as the fuzzy set

$$
\limsup _{k \rightarrow \infty} \tilde{s}_{k}=M\left(\left\{U_{\alpha}\right\}_{\alpha \in] 0,1]}\right) .
$$

The limit of $\left\{\tilde{s}_{k}\right\}_{k \in \mathbb{N}}$ is said to exist if $\liminf _{k \rightarrow \infty} \tilde{s}_{k}=\lim \sup _{k \rightarrow \infty} \tilde{s}_{k}$, and its limit is defined as the fuzzy set

$$
\lim _{k \rightarrow \infty} \tilde{s}_{k}=\liminf _{k \rightarrow \infty} \tilde{s}_{k}=\limsup _{k \rightarrow \infty} \tilde{s}_{k}
$$

For crisp sets $S_{k} \subset \mathbb{R}^{n}, k \in \mathbb{N}$, we set $L=\liminf _{k \rightarrow \infty} S_{k}, U=\limsup _{k \rightarrow \infty} S_{k}$, and set $T=\lim _{k \rightarrow \infty} S_{k}$ if the limit of $\left\{S_{k}\right\}_{k \in \mathbb{N}}$ exists. Then it can be seen that $\liminf _{k \rightarrow \infty} c_{S_{k}}=c_{L}$, $\lim \sup _{k \rightarrow \infty} c_{S_{k}}=c_{U}$, and that $\lim _{k \rightarrow \infty} c_{S_{k}}=c_{T}$ if the limit of $\left\{S_{k}\right\}_{k \in \mathbb{N}}$ exists. Thus, the lower limit, upper limit, and limit for sequences of fuzzy sets are generalizations of the crisp ones.

Example 2.1 Let $\tilde{s}, \tilde{t} \in \mathcal{F}(\mathbb{R})$ be fuzzy sets defined as

$$
\tilde{s}(x)=\left\{\begin{array}{ll}
\max \{-|x|+1,0\} & \text { if } x \neq 0, \\
0 & \text { if } x=0,
\end{array} \quad \tilde{t}(x)=\max \{-|x|+1,0\}\right.
$$


for each $x \in \mathbb{R}$, and let $\left\{\tilde{s}_{k}\right\}_{k \in \mathbb{N}},\left\{\tilde{t}_{k}\right\}_{\in \mathbb{N}},\left\{\tilde{u}_{k}\right\}_{k \in \mathbb{N}} \subset \mathcal{F}(\mathbb{R})$ be sequences of fuzzy sets defined as

$$
\tilde{s}_{k}=\tilde{s}, \quad \tilde{t}_{k}=\tilde{t}, \quad \tilde{u}_{k}= \begin{cases}\tilde{s} & \text { if } k \text { is odd } \\ \tilde{t} & \text { if } k \text { is even }\end{cases}
$$

for each $k \in \mathbb{N}$. In this case, we have $\lim _{k}\left[\tilde{s}_{k}\right]_{\alpha}=\lim _{k}\left[\tilde{t}_{k}\right]_{\alpha}=\lim _{k}\left[\tilde{u}_{k}\right]_{\alpha}=[\alpha-1,1-\alpha]$ for $\alpha \in$ ]0,1[, and $\lim _{k}\left[\tilde{s}_{k}\right]_{1}=\liminf _{k}\left[\tilde{u}_{k}\right]_{1}=\emptyset$ and $\lim _{k}\left[\tilde{t}_{k}\right]_{1}=\lim \sup _{k}\left[\tilde{u}_{k}\right]_{1}=\{0\}$. Thus, it follows that $\lim _{k} \tilde{s}_{k}=\lim _{k} \tilde{t}_{k}=\lim _{k} \tilde{u}_{k}=\tilde{t}$. Therefore, we obtain the following statements.

(i) $\liminf _{k} \tilde{s}_{k}=\liminf _{k} \tilde{t}_{k}$, while $\liminf _{k}\left[\tilde{s}_{k}\right]_{1} \neq \liminf _{k}\left[\tilde{t}_{k}\right]_{1}$.

(ii) $\limsup \sup _{k} \tilde{s}_{k}=\limsup \sup _{k} \tilde{t}_{k}$, while $\lim \sup _{k}\left[\tilde{s}_{k}\right]_{1} \neq \lim \sup _{k}\left[\tilde{t}_{k}\right]_{1}$.

(iii) $\liminf \tilde{u}_{k} \tilde{u}_{k}=\lim \sup _{k} \tilde{u}_{k}$, while $\liminf _{k}\left[\tilde{u}_{k}\right]_{1} \neq \lim \sup _{k}\left[\tilde{u}_{k}\right]_{1}$.

(iv) $\lim _{k} \tilde{s}_{k}=\lim _{k} \tilde{t}_{k}=\lim _{k} \tilde{u}_{k}$, while $\lim _{k}\left[\tilde{s}_{k}\right]_{1} \neq \lim _{k}\left[\tilde{t}_{k}\right]_{1}$ and there does not exist $\lim _{k}\left[\tilde{u}_{k}\right]_{1}$.

From Definition 1.1 and Propositions 1.3, 1.5, and 1.11, the following Proposition 2.1 can be obtained.

Proposition 2.1 Let $\left\{\tilde{s}_{k}\right\}_{k \in \mathbb{N}} \subset \mathcal{F}\left(\mathbb{R}^{n}\right)$, and let $L_{\alpha}=\liminf _{k}\left[\tilde{s}_{k}\right]_{\alpha}, U_{\alpha}=\lim \sup _{k}\left[\tilde{s}_{k}\right]_{\alpha}$ for each $\alpha \in] 0,1]$. Then the following statements hold.

(i) $\left\{L_{\alpha}\right\}_{\alpha \in] 0,1]},\left\{U_{\alpha}\right\}_{\alpha \in] 0,1]} \in \mathcal{S}\left(\mathbb{R}^{n}\right)$.

(ii) $L_{\alpha} \subset U_{\alpha}$ for $\left.\left.\alpha \in\right] 0,1\right]$.

(iii) $\liminf \tilde{s}_{k} \leq \limsup \tilde{s u p}_{k} \tilde{s}_{k}$.

(iv) $L_{\alpha}, U_{\alpha} \in \mathcal{C}\left(\mathbb{R}^{n}\right)$ for $\left.\left.\alpha \in\right] 0,1\right]$.

(v) Let $\alpha \in] 0,1]$, and assume that $\left[\tilde{s}_{k}\right]_{\alpha} \in \mathcal{K}\left(\mathbb{R}^{n}\right), k \in \mathbb{N}$. Then $L_{\alpha} \in \mathcal{K}\left(\mathbb{R}^{n}\right)$.

The following Proposition 2.2(i) is a fuzzified one of Proposition 1.3(i), (iii). The following Proposition 2.2(ii) is a fuzzified one of Proposition 1.5. They can be derived from Propositions 1.3, 1.12, and 2.1.

\section{Proposition 2.2}

(i) Let $\left\{\tilde{s}_{k}\right\}_{k \in \mathbb{N}} \subset \mathcal{F}\left(\mathbb{R}^{n}\right)$. Then $\liminf \tilde{s}_{k}, \tilde{s}_{k}, \lim \sup _{k} \tilde{s}_{k} \in \mathcal{C} \mathcal{F}\left(\mathbb{R}^{n}\right)$. If $\tilde{s}_{k}=\tilde{u}, k \in \mathbb{N}$ for some $\tilde{u} \in \mathcal{F}\left(\mathbb{R}^{n}\right)$, then $\lim _{k} \tilde{s}_{k}=\operatorname{cl}(\tilde{u})$.

(ii) Let $\left\{\tilde{s}_{k}\right\}_{k \in \mathbb{N}} \subset \mathcal{K} \mathcal{F}\left(\mathbb{R}^{n}\right)$. Then $\liminf _{k} \tilde{s}_{k} \in \mathcal{C K \mathcal { F }}\left(\mathbb{R}^{n}\right)$.

The following Proposition 2.3 is a fuzzified one of Proposition 1.1. It can be derived from the decomposition theorem and Propositions 1.1, 1.15, and 1.16.

Proposition 2.3 Let $\left\{\tilde{s}_{k}\right\}_{k \in \mathbb{N}} \subset \mathcal{F}\left(\mathbb{R}^{n}\right)$. Then

$$
\liminf _{k} \tilde{s}_{k}=\bigwedge_{N \in \mathcal{N}_{\infty}^{\sharp}} \operatorname{cl}\left(\bigvee_{k \in N} \tilde{s}_{k}\right) \text { and } \underset{k}{\limsup \tilde{s}_{k}}=\bigwedge_{N \in \mathcal{N}_{\infty}} \operatorname{cl}\left(\bigvee_{k \in N} \tilde{s}_{k}\right)
$$

From Propositions 1.1, 1.15, 1.16, and 2.3, the following Proposition 2.4 can be obtained.

Proposition 2.4 Let $\left\{S_{\alpha}^{(k)}\right\}_{\alpha \in] 0,1]} \in \mathcal{S}\left(\mathbb{R}^{n}\right), k \in \mathbb{N}$, and let $\tilde{s}_{k}=M\left(\left\{S_{\alpha}^{(k)}\right\}_{\alpha \in] 0,1]}\right), k \in \mathbb{N}$. In addition, let $L_{\alpha}=\liminf _{k} S_{\alpha}^{(k)}, U_{\alpha}=\limsup _{k} S_{\alpha}^{(k)}$ for each $\left.\left.\alpha \in\right] 0,1\right]$. Then $\liminf _{k} \tilde{s}_{k}=$ $M\left(\left\{L_{\alpha}\right\}_{\alpha \in] 0,1]}\right)$ and $\lim \sup _{k} \tilde{s}_{k}=M\left(\left\{U_{\alpha}\right\}_{\alpha \in] 0,1]}\right)$. 
The following Proposition 2.5 is a fuzzified one of Proposition 1.3(ii). It can be derived from Propositions 1.3 and 2.4.

Proposition 2.5 Let $\left\{\tilde{s}_{k}\right\}_{k \in \mathbb{N}},\left\{\tilde{t}_{k}\right\}_{k \in \mathbb{N}} \in \mathcal{F}\left(\mathbb{R}^{n}\right)$, and assume that $\operatorname{cl}\left(\tilde{s}_{k}\right)=\operatorname{cl}\left(\tilde{t}_{k}\right), k \in \mathbb{N}$. Then $\liminf _{k} \tilde{s}_{k}=\liminf \tilde{t}_{k}$ and $\lim \sup _{k} \tilde{s}_{k}=\limsup \sup _{k} \tilde{t}_{k}$.

The following Proposition 2.6 is a fuzzified one of Proposition 1.2. It can be derived from Propositions 1.2, 1.11, 1.15, and 1.16.

Proposition 2.6 For $\left\{\tilde{s}_{k}\right\}_{k \in \mathbb{N}},\left\{\tilde{s}_{k}^{1}\right\}_{k \in \mathbb{N}},\left\{\tilde{s}_{k}^{2}\right\}_{k \in \mathbb{N}} \subset \mathcal{F}\left(\mathbb{R}^{n}\right)$, the following statements hold.

(i) If $\tilde{s}_{k} \nearrow$ (which means that $\left.\tilde{s}_{k} \leq \tilde{s}_{k+1} \leq \cdots\right)$, then $\lim _{k} \tilde{s}_{k}=\operatorname{cl}\left(\bigvee_{k \in \mathbb{N}} \tilde{s}_{k}\right)$.

(ii) If $\tilde{s}_{k} \searrow$ (which means that $\tilde{s}_{k} \geq \tilde{s}_{k+1} \geq \cdots$ ), then $\lim _{k} \tilde{s}_{k}=\bigwedge_{k \in \mathbb{N}} \operatorname{cl}\left(\tilde{s}_{k}\right)$.

(iii) If $\tilde{s}_{k}^{1} \leq \tilde{s}_{k} \leq \tilde{s}_{k}^{2}, k \in \mathbb{N}$ and $\liminf _{k} \tilde{s}_{k}^{1}=\liminf _{k} \tilde{s}_{k}^{2}$, then $\liminf _{k} \tilde{s}_{k}=\liminf _{k} \tilde{s}_{k}^{1}=\liminf _{k} \tilde{s}_{k}^{2}$.

(iv) If $\tilde{s}_{k}^{1} \leq \tilde{s}_{k} \leq \tilde{s}_{k}^{2}, k \in \mathbb{N}$ and $\lim \sup _{k} \tilde{s}_{k}^{1}=\lim \sup _{k} \tilde{s}_{k}^{2}$, then $\lim \sup _{k} \tilde{s}_{k}=\lim \sup _{k} \tilde{s}_{k}^{1}=\lim \sup _{k} \tilde{s}_{k}^{2}$.

(v) If $\tilde{s}_{k}^{1} \leq \tilde{s}_{k} \leq \tilde{s}_{k}^{2}, k \in \mathbb{N}$ and $\lim _{k} \tilde{s}_{k}^{1}=\lim _{k} \tilde{s}_{k}^{2}$, then $\lim _{k} \tilde{s}_{k}=\lim _{k} \tilde{s}_{k}^{1}=\lim _{k} \tilde{s}_{k}^{2}$.

The following Proposition 2.7 is a fuzzified one of Proposition 1.6. It can be derived from Definition 1.1 and Proposition 1.11.

Proposition 2.7 Let $\left\{\tilde{s}_{k}\right\}_{k \in \mathbb{N}} \subset \mathcal{F}\left(\mathbb{R}^{n}\right)$, and let $\tilde{s}=\lim _{k \rightarrow \infty} \tilde{s}_{k}$. In addition, let $N \in \mathcal{N}_{\infty}^{\sharp}$. Then $\tilde{s}=\lim _{k \rightarrow \infty} \infty \tilde{s}_{k}$.

Throughout the rest of this section, let $X \subset \mathbb{R}^{n}$ be a compact set, and let

$$
\mathcal{F}^{\prime}\left(\mathbb{R}^{n}\right)=\left\{\tilde{s} \in \mathcal{C} \mathcal{F}\left(\mathbb{R}^{n}\right): \sup _{\mathbf{x} \in \mathbb{R}^{n}} \tilde{s}(\mathbf{x})=1 \text { and } \tilde{s}(\mathbf{x})=0, \mathbf{x} \notin X\right\} .
$$

For $\tilde{s} \in \mathcal{F}^{\prime}\left(\mathbb{R}^{n}\right)$ and $\alpha \in[0,1]$, we set

$$
[[\tilde{s}]]_{\alpha}= \begin{cases}{[\tilde{s}]_{\alpha}} & \text { if } \alpha \in] 0,1] \\ \operatorname{cl}\left(\left\{\mathbf{x} \in \mathbb{R}^{n}: \tilde{s}(\mathbf{x})>0\right\}\right) & \text { if } \alpha=0\end{cases}
$$

Let $\left\{\tilde{s}_{k}\right\}_{k \in \mathbb{N}} \subset \mathcal{F}^{\prime}\left(\mathbb{R}^{n}\right)$, and let $\tilde{s} \in \mathcal{F}^{\prime}\left(\mathbb{R}^{n}\right)$. Then $\left\{\tilde{s}_{k}\right\}_{k \in \mathbb{N}}$ is said to converge to $\tilde{s}$ in the sense of Yoshida et al. [9] if $\rho\left(\left[\left[\tilde{s}_{k}\right]\right]_{\alpha},[[\tilde{s}]]_{\alpha}\right) \rightarrow 0$ for any $\alpha \in[0,1]$ except for at most countable many $\alpha$, where $\rho\left(\left[\left[\tilde{s}_{k}\right]\right]_{\alpha},[[\tilde{s}]]_{\alpha}\right)$ is the Hausdorff distance between $\left[\left[\tilde{s}_{k}\right]\right]_{\alpha}$ and $[[\tilde{s}]]_{\alpha}$ defined in Definition 1.2. From Proposition 1.4, $\rho\left(\left[\left[\tilde{s}_{k}\right]\right]_{\alpha},[[\tilde{s}]]_{\alpha}\right) \rightarrow 0$ if and only if $\lim _{k}\left[\left[\tilde{s}_{k}\right]\right]_{\alpha}=$ $[[\tilde{s}]]_{\alpha}$ in the sense of Definition 1.1.

The following Proposition 2.8 shows that the concept of the convergence for sequences of fuzzy sets in the sense of Definition 2.1 is weaker than that in the sense of Yoshida et al. [9].

Proposition 2.8 Let $\left\{\tilde{s}_{k}\right\}_{k \in \mathbb{N}} \subset \mathcal{F}^{\prime}\left(\mathbb{R}^{n}\right)$, and let $\tilde{s} \in \mathcal{F}^{\prime}\left(\mathbb{R}^{n}\right)$. If $\left\{\tilde{s}_{k}\right\}_{k \in \mathbb{N}}$ converges to $\tilde{s}$ in the sense of Yoshida et al. [9], then $\lim _{k} \tilde{s}_{k}=\tilde{s}$ in the sense of Definition 2.1.

Proof $\left\{\tilde{s}_{k}\right\}_{k \in \mathbb{N}}$ converges to $\tilde{s}$ in the sense of Yoshida et al. [9] if and only if $\lim _{k}\left[\left[\tilde{s}_{k}\right]\right]_{\alpha}=$ $[[\tilde{s}]]_{\alpha}$ in the sense of Definition 1.1 for any $\alpha \in[0,1]$ except for at most countable many $\alpha$. 
Then it follows that $\lim _{k}\left[\tilde{s}_{k}\right]_{\alpha}=\liminf { }_{k}\left[\tilde{s}_{k}\right]_{\alpha}=\lim \sup _{k}\left[\tilde{s}_{k}\right]_{\alpha}=[\tilde{s}]_{\alpha}$ for any $\left.\left.\alpha \in\right] 0,1\right]$ except for at most countable many $\alpha$. From Proposition 1.17 and the decomposition theorem, we have $\tilde{s}=\lim _{k} \tilde{s}_{k}=\liminf _{k} \tilde{s}_{k}=\lim \sup _{k} \tilde{s}_{k}$ in the sense of Definition 2.1.

\section{Limits of fuzzy set-valued mappings}

In this section, we propose the definitions of limits of fuzzy set-valued mappings, and investigate their properties.

For a fuzzy set-valued mapping $\tilde{F}: \mathbb{R}^{n} \rightarrow \mathcal{F}\left(\mathbb{R}^{m}\right)$ and $\left.\left.\alpha \in\right] 0,1\right]$, we define the crisp setvalued mapping $F_{\alpha}: \mathbb{R}^{n} \rightsquigarrow \mathbb{R}^{m}$ as

$$
F_{\alpha}(\mathbf{x})=[\tilde{F}(\mathbf{x})]_{\alpha}
$$

for each $\mathbf{x} \in \mathbb{R}^{n}$.

A fuzzy set-valued mapping $\tilde{F}: \mathbb{R}^{n} \rightarrow \mathcal{F}\left(\mathbb{R}^{m}\right)$ is said to be closed-valued, convex-valued, or closed convex-valued if $\tilde{F}(\mathbf{x}) \in \mathcal{C} \mathcal{F}\left(\mathbb{R}^{m}\right), \tilde{F}(\mathbf{x}) \in \mathcal{K} \mathcal{F}\left(\mathbb{R}^{m}\right)$, or $\tilde{F}(\mathbf{x}) \in \mathcal{C K} \mathcal{F}\left(\mathbb{R}^{m}\right)$ for any $\mathbf{x} \in \mathbb{R}^{n}$, respectively.

The following Definition 3.1 is a fuzzified one of Definition 1.3 (see Proposition 3.3 which is mentioned later).

Definition 3.1 Let $\tilde{F}: \mathbb{R}^{n} \rightarrow \mathcal{F}\left(\mathbb{R}^{m}\right)$, and let $\overline{\mathbf{x}} \in \mathbb{R}^{n}$. In addition, let

$$
L_{\alpha}(\overline{\mathbf{x}})=\liminf _{\mathbf{x} \rightarrow \overline{\mathbf{x}}} F_{\alpha}(\mathbf{x}), \quad U_{\alpha}(\overline{\mathbf{x}})=\limsup _{\mathbf{x} \rightarrow \overline{\mathbf{x}}} F_{\alpha}(\mathbf{x})
$$

for each $\alpha \in] 0,1]$. The lower limit of $\tilde{F}$ when $\mathbf{x} \rightarrow \overline{\mathbf{x}}$ is defined as the fuzzy set

$$
\liminf _{\mathbf{x} \rightarrow \mathbf{x}} \tilde{F}(\mathbf{x})=M\left(\left\{L_{\alpha}(\overline{\mathbf{x}})\right\}_{\alpha \in] 0,1]}\right)
$$

and the upper limit of $\tilde{F}$ when $\mathbf{x} \rightarrow \overline{\mathbf{x}}$ is defined as the fuzzy set

$$
\underset{\mathbf{x} \rightarrow \overline{\mathbf{x}}}{\limsup } \tilde{F}(\mathbf{x})=M\left(\left\{U_{\alpha}(\overline{\mathbf{x}})\right\}_{\alpha \in] 0,1]}\right) .
$$

The limit of $\tilde{F}$ when $\mathbf{x} \rightarrow \overline{\mathbf{x}}$ is said to exist if $\liminf _{\mathbf{x} \rightarrow \overline{\mathbf{x}}} \tilde{F}(\mathbf{x})=\lim \sup _{\mathbf{x} \rightarrow \overline{\mathbf{x}}} \tilde{F}(\mathbf{x})$, and its limit is defined as the fuzzy set

$$
\lim _{\mathbf{x} \rightarrow \overline{\mathbf{x}}} \tilde{F}(\mathbf{x})=\liminf _{\mathbf{x} \rightarrow \overline{\mathbf{x}}} \tilde{F}(\mathbf{x})=\limsup _{\mathbf{x} \rightarrow \overline{\mathbf{x}}} \tilde{F}(\mathbf{x})
$$

For a crisp set-valued mapping $F: \mathbb{R}^{n} \rightsquigarrow \mathbb{R}^{m}$ and $\overline{\mathbf{x}} \in \mathbb{R}^{n}$, let $L(\overline{\mathbf{x}})=\liminf _{\mathbf{x} \rightarrow \overline{\mathbf{x}}} F(\mathbf{x})$, $U(\overline{\mathbf{x}})=\limsup _{\mathbf{x} \rightarrow \overline{\mathbf{x}}} F(\mathbf{x})$, and let $T(\overline{\mathbf{x}})=\lim _{\mathbf{x} \rightarrow \overline{\mathbf{x}}} F(\mathbf{x})$ if the limit of $F$ when $\mathbf{x} \rightarrow \overline{\mathbf{x}}$ exists. Then $\liminf _{\mathbf{x} \rightarrow \overline{\mathbf{x}}} c_{F(\mathbf{x})}=c_{L(\overline{\mathbf{x}})}, \limsup _{\mathbf{x} \rightarrow \overline{\mathbf{x}}} c_{F(\mathbf{x})}=c_{U(\overline{\mathbf{x}})}$, and $\lim _{\mathbf{x} \rightarrow \overline{\mathbf{x}}} c_{F(\mathbf{x})}=c_{T(\overline{\mathbf{x}})}$ if the limit of $F$ when $\mathbf{x} \rightarrow \overline{\mathbf{x}}$ exists. Thus, the lower limit, upper limit, and limit for fuzzy set-valued mappings are generalizations of the crisp ones.

Example 3.1 Let $\tilde{s}, \tilde{t}, \tilde{\emptyset} \in \mathcal{F}(\mathbb{R})$ be fuzzy sets defined as

$$
\tilde{s}(x)=\left\{\begin{array}{ll}
\max \{-|x|+1,0\} & \text { if } x \neq 0, \\
0 & \text { if } x=0,
\end{array} \quad \tilde{t}(x)=\max \{-|x|+1,0\}, \quad \tilde{\emptyset}(x)=0\right.
$$


for each $x \in \mathbb{R}$, and let $\tilde{F}: \mathbb{R} \rightarrow \mathcal{F}(\mathbb{R})$ be a fuzzy set-valued mapping defined as

$$
\tilde{F}(x)= \begin{cases}\tilde{s} & \text { if } x \in B(1, \varepsilon) \cup(B(3, \varepsilon) \cap \mathbb{Q}), \\ \tilde{t} & \text { if } x \in B(2, \varepsilon) \cup(B(3, \varepsilon) \backslash \mathbb{Q}), \\ \tilde{\emptyset} & \text { otherwise }\end{cases}
$$

for each $x \in \mathbb{R}$, where $\mathbb{Q}$ is the set of all rational numbers, and $\varepsilon \in] 0, \frac{1}{2}[$, and $B(\bar{x}, \varepsilon)=\{x \in$ $\mathbb{R}:|x-\bar{x}|<\varepsilon\}$ for $\bar{x} \in \mathbb{R}$. Since

$$
F_{\alpha}(x)= \begin{cases}{[\alpha-1,0[\cup] 0,1-\alpha]} & \text { if } x \in B(1, \varepsilon) \cup(B(3, \varepsilon) \cap \mathbb{Q}), \\ {[\alpha-1,1-\alpha]} & \text { if } x \in B(2, \varepsilon) \cup(B(3, \varepsilon) \backslash \mathbb{Q}), \\ \emptyset & \text { otherwise }\end{cases}
$$

for each $x \in \mathbb{R}$ when $\alpha \in] 0,1[$, and

$$
F_{1}(x)= \begin{cases}\{0\} & \text { if } x \in B(2, \varepsilon) \cup(B(3, \varepsilon) \backslash \mathbb{Q}) \\ \emptyset & \text { otherwise }\end{cases}
$$

for each $x \in \mathbb{R}$, we have $\lim _{x \rightarrow 1} F_{\alpha}(x)=\lim _{x \rightarrow 2} F_{\alpha}(x)=\lim _{x \rightarrow 3} F_{\alpha}(x)=[\alpha-1,1-\alpha]$ for $\alpha \in] 0,1\left[\right.$, and $\lim _{x \rightarrow 1} F_{1}(x)=\liminf _{x \rightarrow 3} F_{1}(x)=\emptyset$ and $\lim _{x \rightarrow 2} F_{1}(x)=\limsup _{x \rightarrow 3} F_{1}(x)=\{0\}$. Thus, it follows that $\lim _{x \rightarrow 1} \tilde{F}(x)=\lim _{x \rightarrow 2} \tilde{F}(x)=\lim _{x \rightarrow 3} \tilde{F}(x)=\tilde{t}$. Therefore, we obtain the following statements.

(i) $\liminf _{x \rightarrow 1} \tilde{F}(x)=\liminf _{x \rightarrow 2} \tilde{F}(x)$, while $\liminf _{x \rightarrow 1} F_{1}(x) \neq \liminf _{x \rightarrow 2} F_{1}(x)$.

(ii) $\lim \sup _{x \rightarrow 1} \tilde{F}(x)=\lim \sup _{x \rightarrow 2} \tilde{F}(x)$, while $\lim \sup _{x \rightarrow 1} F_{1}(x) \neq \limsup _{x \rightarrow 2} F_{1}(x)$.

(iii) $\liminf _{x \rightarrow 3} \tilde{F}(x)=\limsup _{x \rightarrow 3} \tilde{F}(x)$, while $\liminf _{x \rightarrow 3} F_{1}(x) \neq \limsup _{x \rightarrow 3} F_{1}(x)$.

(iv) $\lim _{x \rightarrow 1} \tilde{F}(x)=\lim _{x \rightarrow 2} \tilde{F}(x)=\lim _{x \rightarrow 3} \tilde{F}(x)$, while $\lim _{x \rightarrow 1} F_{1}(x) \neq \lim _{x \rightarrow 2} F_{1}(x)$ and there does not exist $\lim _{x \rightarrow 3} F_{1}(x)$.

The following Definition 3.2 is a fuzzified one of Definition 1.4.

Definition 3.2 Let $\tilde{F}: \mathbb{R}^{n} \rightarrow \mathcal{F}\left(\mathbb{R}^{m}\right)$, and let $\overline{\mathbf{x}} \in \mathbb{R}^{n}$. The fuzzy set-valued mapping $\tilde{F}$ is said to be lower semicontinuous at $\overline{\mathbf{x}}$ if

$$
\liminf _{\mathbf{x} \rightarrow \overline{\mathbf{x}}} \tilde{F}(\mathbf{x}) \geq \tilde{F}(\overline{\mathbf{x}})
$$

and $\tilde{F}$ is said to be upper semicontinuous at $\overline{\mathbf{x}}$ if

$$
\limsup _{\mathbf{x} \rightarrow \overline{\mathbf{x}}} \tilde{F}(\mathbf{x}) \leq \tilde{F}(\overline{\mathbf{x}})
$$

The fuzzy set-valued mapping $\tilde{F}$ is said to be continuous at $\overline{\mathbf{x}}$ if $\tilde{F}$ is both lower and upper semicontinuous at $\overline{\mathbf{x}}$, that is,

$$
\lim _{\mathbf{x} \rightarrow \overline{\mathbf{x}}} \tilde{F}(\mathbf{x})=\tilde{F}(\overline{\mathbf{x}}) .
$$

Example 3.2 Let $\tilde{s}, \tilde{t} \in \mathcal{F}(\mathbb{R})$ be fuzzy sets defined as $\tilde{s}(x)=\max \left\{-|x|+\frac{1}{2}, 0\right\}$ and $\tilde{t}(x)=$ $\max \{-|x|+1,0\}$ for each $x \in \mathbb{R}$, and let $\tilde{F}, \tilde{G}: \mathbb{R} \rightarrow \mathcal{F}(\mathbb{R})$ be fuzzy set-valued mappings 
defined as

$$
\tilde{F}(x)=\left\{\begin{array}{ll}
\tilde{s} & \text { if } x \leq 0, \\
\tilde{t} & \text { if } x>0,
\end{array} \quad \tilde{G}(x)= \begin{cases}\tilde{s} & \text { if } x<0 \\
\tilde{t} & \text { if } x \geq 0\end{cases}\right.
$$

for each $x \in \mathbb{R}$. Since $\liminf _{x \rightarrow 0} \tilde{F}(x)=\tilde{s} \geq \tilde{s}=\tilde{F}(0)$ and $\limsup _{x \rightarrow 0} \tilde{F}(x)=\tilde{t} \not \leq \tilde{s}=\tilde{F}(0), \tilde{F}$ is lower semicontinuous at 0 but not upper semicontinuous at 0 . Since $\lim \sup _{x \rightarrow 0} \tilde{G}(x)=\tilde{t} \leq$ $\tilde{t}=\tilde{G}(0)$ and $\liminf _{x \rightarrow 0} \tilde{G}(x)=\tilde{s} \nsupseteq \tilde{t}=\tilde{G}(0), \tilde{G}$ is upper semicontinuous at 0 but not lower semicontinuous at 0 .

From Definition 1.3 and Propositions 1.3, 1.5, and 1.11, the following Proposition 3.1 can be obtained.

Proposition 3.1 Let $\tilde{F}: \mathbb{R}^{n} \rightarrow \mathcal{F}\left(\mathbb{R}^{m}\right)$, and let $\overline{\mathbf{x}} \in \mathbb{R}^{n}$. In addition, let

$$
L_{\alpha}(\overline{\mathbf{x}})=\liminf _{\mathbf{x} \rightarrow \overline{\mathbf{x}}} F_{\alpha}(\mathbf{x}), \quad U_{\alpha}(\overline{\mathbf{x}})=\limsup _{\mathbf{x} \rightarrow \overline{\mathbf{x}}} F_{\alpha}(\mathbf{x})
$$

for each $\alpha \in] 0,1]$. Then the following statements hold.

(i) $\left\{L_{\alpha}(\overline{\mathbf{x}})\right\}_{\alpha \in] 0,1]},\left\{U_{\alpha}(\overline{\mathbf{x}})\right\}_{\alpha \in] 0,1]} \in \mathcal{S}\left(\mathbb{R}^{m}\right)$.

(ii) $L_{\alpha}(\overline{\mathbf{x}}) \subset \operatorname{cl}\left(F_{\alpha}(\overline{\mathbf{x}})\right) \subset U_{\alpha}(\overline{\mathbf{x}})$ for $\left.\left.\alpha \in\right] 0,1\right]$.

(iii) $\liminf _{\mathbf{x} \rightarrow \overline{\mathbf{x}}} \tilde{F}(\mathbf{x}) \leq \operatorname{cl}(\tilde{F}(\overline{\mathbf{x}})) \leq \limsup _{\mathbf{x} \rightarrow \overline{\mathbf{x}}} \tilde{F}(\mathbf{x})$.

(iv) $L_{\alpha}(\overline{\mathbf{x}}) \in \mathcal{C}\left(\mathbb{R}^{m}\right)$ for $\left.\left.\alpha \in\right] 0,1\right]$.

(v) Let $\alpha \in] 0,1]$, and assume that $F_{\alpha}$ is convex-valued. Then $L_{\alpha}(\overline{\mathbf{x}}) \in \mathcal{K}\left(\mathbb{R}^{m}\right)$.

From Propositions 1.12 and 3.1, the following Proposition 3.2 can be obtained.

Proposition 3.2 Let $\tilde{F}: \mathbb{R}^{n} \rightarrow \mathcal{F}\left(\mathbb{R}^{m}\right)$, and let $\overline{\mathbf{x}} \in \mathbb{R}^{n}$. Then the following statements hold.

(i) $\liminf _{\mathbf{x} \rightarrow \overline{\mathbf{x}}} \tilde{F}(\mathbf{x}) \in \mathcal{C} \mathcal{F}\left(\mathbb{R}^{m}\right)$.

(ii) If $\tilde{F}$ is convex-valued, then $\liminf _{\mathbf{x} \rightarrow \overline{\mathbf{x}}} \tilde{F}(\mathbf{x}) \in \mathcal{C K} \mathcal{F}\left(\mathbb{R}^{m}\right)$.

The following Proposition 3.3 shows that Definition 3.1 is a fuzzified one of Definition 1.3. It can be derived from Proposition 1.16.

Proposition 3.3 Let $\tilde{F}: \mathbb{R}^{n} \rightarrow \mathcal{F}\left(\mathbb{R}^{m}\right)$, and let $\overline{\mathbf{x}} \in \mathbb{R}^{n}$. Then

$$
\liminf _{\mathbf{x} \rightarrow \overline{\mathbf{x}}} \tilde{F}(\mathbf{x})=\bigwedge_{\mathbf{x}_{k} \rightarrow \overline{\mathbf{x}}} \liminf _{k} \tilde{F}\left(\mathbf{x}_{k}\right) \quad \text { and } \quad \limsup _{\mathbf{x} \rightarrow \overline{\mathbf{x}}} \tilde{F}(\mathbf{x})=\bigvee_{\mathbf{x}_{k} \rightarrow \overline{\mathbf{x}}} \limsup _{k} \tilde{F}\left(\mathbf{x}_{k}\right)
$$

From Propositions 1.16, 2.4, and 3.3, the following Proposition 3.4 can be obtained.

Proposition 3.4 Let $\left\{S_{\alpha}(\mathbf{x})\right\}_{\alpha \in] 0,1]} \in \mathcal{S}\left(\mathbb{R}^{m}\right), \mathbf{x} \in \mathbb{R}^{n}$, and assume that $\tilde{F}: \mathbb{R}^{n} \rightarrow \mathcal{F}\left(\mathbb{R}^{m}\right)$ is defined as $\tilde{F}(\mathbf{x})=M\left(\left\{S_{\alpha}(\mathbf{x})\right\}_{\alpha \in] 0,1]}\right)$ for each $\mathbf{x} \in \mathbb{R}^{n}$. In addition, let $\overline{\mathbf{x}} \in \mathbb{R}^{n}$, and let $L_{\alpha}(\overline{\mathbf{x}})=\liminf _{\mathbf{x} \rightarrow \overline{\mathbf{x}}} S_{\alpha}(\mathbf{x}), U_{\alpha}(\overline{\mathbf{x}})=\limsup _{\mathbf{x} \rightarrow \overline{\mathbf{x}}} S_{\alpha}(\mathbf{x})$ for each $\left.\left.\alpha \in\right] 0,1\right]$. Then $\liminf _{\mathbf{x} \rightarrow \overline{\mathbf{x}}} \tilde{F}(\mathbf{x})=$ $M\left(\left\{L_{\alpha}(\overline{\mathbf{x}})\right\}_{\alpha \in] 0,1]}\right)$ and $\lim \sup _{\mathbf{x} \rightarrow \overline{\mathbf{x}}} \tilde{F}(\mathbf{x})=M\left(\left\{U_{\alpha}(\overline{\mathbf{x}})\right\}_{\alpha \in] 0,1]}\right)$.

The following Proposition 3.5 is a fuzzified one of Proposition 1.7. It can be derived from Proposition 3.3. 
Proposition 3.5 Let $\tilde{F}: \mathbb{R}^{n} \rightarrow \mathcal{F}\left(\mathbb{R}^{m}\right)$, and let $\overline{\mathbf{x}} \in \mathbb{R}^{n}$. Then $\lim _{\mathbf{x} \rightarrow \overline{\mathbf{x}}} \tilde{F}(\mathbf{x})=\tilde{F}(\overline{\mathbf{x}})$ if and only if $\lim _{k} \tilde{F}\left(\mathbf{x}_{k}\right)=\tilde{F}(\overline{\mathbf{x}})$ for any sequence $\left\{\mathbf{x}_{k}\right\}_{k \in \mathbb{N}} \subset \mathbb{R}^{n}$ such that $\mathbf{x}_{k} \rightarrow \overline{\mathbf{x}}$.

\section{Derivatives of fuzzy set-valued mappings}

In this section, we propose the definition of derivatives of fuzzy set-valued mappings and investigate their properties.

The following Definition 4.1 is a fuzzified one of Definition 1.5.

Definition 4.1 Let $\tilde{s} \in \mathcal{F}\left(\mathbb{R}^{n}\right)$, and let $\mathbf{x}_{0} \in[\tilde{s}]_{1}$. Then the fuzzy set

$$
\tilde{T}\left(\tilde{s} ; \mathbf{x}_{0}\right)=M\left(\left\{T\left([\tilde{s}]_{\alpha} ; \mathbf{x}_{0}\right)\right\}_{\alpha \in] 0,1]}\right)
$$

is called the fuzzy tangent cone or fuzzy contingent cone of $\tilde{s}$ at $\mathbf{x}_{0}$.

Let $S \subset \mathbb{R}^{n}$, and let $\mathbf{x}_{0} \in S$. Then it can be seen that

$$
\tilde{T}\left(c_{S} ; \mathbf{x}_{0}\right)=c_{T\left(S ; \mathbf{x}_{0}\right)} .
$$

Thus, the fuzzy tangent cone is a generalization of the crisp one.

Example 4.1 Let $f: \mathbb{R} \rightarrow \mathbb{R}$ be a function defined as $f(x)=(x+1) x(x-1)=x^{3}-x$ for each $x \in \mathbb{R}$, and let $\tilde{s} \in \mathcal{F}\left(\mathbb{R}^{2}\right)$ be a fuzzy set defined as, for each $\mathbf{x}=(x, y) \in \mathbb{R}^{2}$,

$$
\tilde{s}(\mathbf{x})=\max \left\{-\frac{1}{|f(x)|}|y-f(x)|+1,0\right\}
$$

if $f(x) \neq 0$, and

$$
\tilde{s}(\mathbf{x})= \begin{cases}1 & \text { if } y=0, \\ 0 & \text { if } y \neq 0\end{cases}
$$

if $f(x)=0$. In addition, let $\mathbf{x}_{0}=(0,0) \in[\tilde{s}]_{1}$. For each $\left.\left.\alpha \in\right] 0,1\right]$, since $[\tilde{s}]_{\alpha}=\{(x, y) \in$ $\left.\mathbb{R}^{2}: \min \left\{\alpha\left(x^{3}-x\right),(2-\alpha)\left(x^{3}-x\right)\right\} \leq y \leq \max \left\{\alpha\left(x^{3}-x\right),(2-\alpha)\left(x^{3}-x\right)\right\}\right\}$, it follows that $T\left([\tilde{s}]_{\alpha} ; \mathbf{x}_{0}\right)=\left\{(x, y) \in \mathbb{R}^{2}: \min \{-\alpha x,-(2-\alpha) x\} \leq y \leq \max \{-\alpha x,-(2-\alpha) x\}\right\}$ from Example 1.4. Therefore, for each $\mathbf{x}=(x, y) \in \mathbb{R}^{2}$, we have

$$
\tilde{T}\left(\tilde{s} ; \mathbf{x}_{0}\right)(\mathbf{x})=\max \left\{-\frac{1}{|x|}|y+x|+1,0\right\}
$$

if $x \neq 0$, and

$$
\tilde{T}\left(\tilde{s} ; \mathbf{x}_{0}\right)(\mathbf{x})= \begin{cases}1 & \text { if } y=0 \\ 0 & \text { if } y \neq 0\end{cases}
$$

if $x=0$.

The following Proposition 4.1 is a fuzzified one of Proposition 1.8. It can be derived from Propositions 1.8 and 1.12 . 
Proposition 4.1 Let $\tilde{s} \in \mathcal{F}\left(\mathbb{R}^{n}\right)$, and let $\mathbf{x}_{0} \in[\tilde{s}]_{1}$. Then the following statements hold.

(i) $\tilde{T}\left(\tilde{s} ; \mathbf{x}_{0}\right)$ is a closed fuzzy cone.

(ii) If $\tilde{s} \in \mathcal{K} \mathcal{F}\left(\mathbb{R}^{n}\right)$, then $\tilde{T}\left(\tilde{s} ; \mathbf{x}_{0}\right)$ is a closed convex fuzzy cone.

The following Proposition 4.2 is a fuzzified one of Proposition 1.9. It can be derived from Propositions 1.9 and 1.11.

Proposition 4.2 Let $\tilde{s}, \tilde{t} \in \mathcal{F}\left(\mathbb{R}^{n}\right)$ with $\tilde{s} \leq \tilde{t}$, and let $\mathbf{x}_{0} \in[\tilde{s}]_{1}$. Then $\tilde{T}\left(\tilde{s} ; \mathbf{x}_{0}\right) \leq \tilde{T}\left(\tilde{t} ; \mathbf{x}_{0}\right)$.

The following Definition 4.2 is a fuzzified one of the graph for crisp set-valued mappings.

Definition 4.2 Let $\tilde{F}: \mathbb{R}^{n} \rightarrow \mathcal{F}\left(\mathbb{R}^{m}\right)$. The fuzzy set $\operatorname{Graph}(\tilde{F}) \in \mathcal{F}\left(\mathbb{R}^{n} \times \mathbb{R}^{m}\right)$ defined as

$$
\operatorname{Graph}(\tilde{F})(\mathbf{x}, \mathbf{y})=\tilde{F}(\mathbf{x})(\mathbf{y})
$$

for each $(\mathbf{x}, \mathbf{y}) \in \mathbb{R}^{n} \times \mathbb{R}^{m}$ is called the fuzzy graph of $\tilde{F}$.

From Definition 4.2, it can be seen that

$$
[\operatorname{Graph}(\tilde{F})]_{\alpha}=\operatorname{Graph}\left(F_{\alpha}\right)
$$

for $\alpha \in] 0,1]$.

Let $F: \mathbb{R}^{n} \rightsquigarrow \mathbb{R}^{m}$, and assume that $\tilde{F}: \mathbb{R}^{n} \rightarrow \mathcal{F}\left(\mathbb{R}^{m}\right)$ is defined as $\tilde{F}(\mathbf{x})=c_{F(\mathbf{x})}$ for each $\mathbf{x} \in \mathbb{R}^{n}$. Then it follows that

$$
\operatorname{Graph}(\tilde{F})(\mathbf{x}, \mathbf{y})=\tilde{F}(\mathbf{x})(\mathbf{y})=c_{F(\mathbf{x})}(\mathbf{y})=c_{\mathrm{Graph}(F)}(\mathbf{x}, \mathbf{y})
$$

for each $(\mathbf{x}, \mathbf{y}) \in \mathbb{R}^{n} \times \mathbb{R}^{m}$. Thus, the fuzzy graph for fuzzy set-valued mappings is a generalization of the crisp one.

The following Definition 4.3 is a fuzzified one of Definition 1.6.

Definition 4.3 Let $\tilde{F}: \mathbb{R}^{n} \rightarrow \mathcal{F}\left(\mathbb{R}^{m}\right)$, and let $\left(\mathbf{x}_{0}, \mathbf{y}_{0}\right) \in[\operatorname{Graph}(\tilde{F})]_{1}$. Then the fuzzy setvalued mapping $D \tilde{F}\left(\mathbf{x}_{0}, \mathbf{y}_{0}\right): \mathbb{R}^{n} \rightarrow \mathcal{F}\left(\mathbb{R}^{m}\right)$ such that

$$
\operatorname{Graph}\left(D \tilde{F}\left(\mathbf{x}_{0}, \mathbf{y}_{0}\right)\right)=\tilde{T}\left(\operatorname{Graph}(\tilde{F}) ;\left(\mathbf{x}_{0}, \mathbf{y}_{0}\right)\right)
$$

is called the fuzzy contingent derivative of $\tilde{F}$ at $\left(\mathbf{x}_{0}, \mathbf{y}_{0}\right)$.

From Definition 4.3, it can be seen that

$$
D \tilde{F}\left(\mathbf{x}_{0}, \mathbf{y}_{0}\right)(\mathbf{u})(\mathbf{v})=\operatorname{Graph}\left(D \tilde{F}\left(\mathbf{x}_{0}, \mathbf{y}_{0}\right)\right)(\mathbf{u}, \mathbf{v})=\tilde{T}\left(\operatorname{Graph}(\tilde{F}) ;\left(\mathbf{x}_{0}, \mathbf{y}_{0}\right)\right)(\mathbf{u}, \mathbf{v})
$$

for each $\mathbf{u} \in \mathbb{R}^{n}$ and each $\mathbf{v} \in \mathbb{R}^{m}$.

Let $F: \mathbb{R}^{n} \rightsquigarrow \mathbb{R}^{m}$, and let $\left(\mathbf{x}_{0}, \mathbf{y}_{0}\right) \in \operatorname{Graph}(F)$. Assume that $\tilde{F}: \mathbb{R}^{n} \rightarrow \mathcal{F}\left(\mathbb{R}^{m}\right)$ is defined as $\tilde{F}(\mathbf{x})=c_{F(\mathbf{x})}$ for each $\mathbf{x} \in \mathbb{R}^{n}$. Then it follows that

$$
\operatorname{Graph}(\tilde{F})\left(\mathbf{x}_{0}, \mathbf{y}_{0}\right)=c_{\operatorname{Graph}(F)}\left(\mathbf{x}_{0}, \mathbf{y}_{0}\right)=1
$$


that is, $\left(\mathbf{x}_{0}, \mathbf{y}_{0}\right) \in[\operatorname{Graph}(\tilde{F})]_{1}$, and that

$$
\begin{aligned}
\operatorname{Graph}\left(D \tilde{F}\left(\mathbf{x}_{0}, \mathbf{y}_{0}\right)\right) & =\tilde{T}\left(\operatorname{Graph}(\tilde{F}) ;\left(\mathbf{x}_{0}, \mathbf{y}_{0}\right)\right)=\tilde{T}\left(c_{\operatorname{Graph}(F)} ;\left(\mathbf{x}_{0}, \mathbf{y}_{0}\right)\right) \\
& =c_{T\left(\operatorname{Graph}(F) ;\left(\mathbf{x}_{0}, \mathbf{y}_{0}\right)\right)}=c_{\operatorname{Graph}\left(D F\left(\mathbf{x}_{0}, \mathbf{y}_{0}\right)\right)},
\end{aligned}
$$

that is,

$$
\operatorname{Graph}\left(D \tilde{F}\left(\mathbf{x}_{0}, \mathbf{y}_{0}\right)\right)=c_{\operatorname{Graph}\left(D F\left(\mathbf{x}_{0}, \mathbf{y}_{0}\right)\right)}
$$

Thus, the fuzzy contingent derivative for fuzzy set-valued mappings is a generalization of the crisp one.

Example 4.2 Let $f: \mathbb{R} \rightarrow \mathbb{R}$ be the function defined in Example 4.1, and let $\tilde{F}: \mathbb{R} \rightarrow \mathcal{F}(\mathbb{R})$ be a fuzzy set-valued mapping defined as, for each $x \in \mathbb{R}$,

$$
\tilde{F}(x)(y)=\max \left\{-\frac{1}{|f(x)|}|y-f(x)|+1,0\right\}
$$

for each $y \in \mathbb{R}$ if $f(x) \neq 0$, and

$$
\tilde{F}(x)(y)= \begin{cases}1 & \text { if } y=0 \\ 0 & \text { if } y \neq 0\end{cases}
$$

for each $y \in \mathbb{R}$ if $f(x)=0$. In addition, let $\tilde{s} \in \mathcal{F}\left(\mathbb{R}^{2}\right)$ be the fuzzy set defined in Example 4.1. Then it follows that $\operatorname{Graph}(\tilde{F})=\tilde{s}$ and $(0,0) \in[\operatorname{Graph}(\tilde{F})]_{1}$. From Example 4.1, we have, for each $u \in \mathbb{R}$ and each $v \in \mathbb{R}$,

$$
D \tilde{F}(0,0)(u)(v)=\max \left\{-\frac{1}{|u|}|v+u|+1,0\right\}
$$

if $u \neq 0$, and

$$
D \tilde{F}(0,0)(0)(v)= \begin{cases}1 & \text { if } v=0 \\ 0 & \text { if } v \neq 0\end{cases}
$$

Proposition 4.3 Let $\tilde{F}: \mathbb{R}^{n} \rightarrow \mathcal{F}\left(\mathbb{R}^{m}\right)$, and let $\left(\mathbf{x}_{0}, \mathbf{y}_{0}\right) \in[\operatorname{Graph}(\tilde{F})]_{1}$. Then $D \tilde{F}\left(\mathbf{x}_{0}, \mathbf{y}_{0}\right)(\mathbf{u})=$ $M\left(\left\{D F_{\alpha}\left(\mathbf{x}_{0}, \mathbf{y}_{0}\right)(\mathbf{u})\right\}_{\alpha \in] 0,1]}\right)$ for any $\mathbf{u} \in \mathbb{R}^{n}$.

Proof Fix any $\mathbf{u} \in \mathbb{R}^{n}$ and any $\mathbf{v} \in \mathbb{R}^{m}$. Then we have

$$
\begin{aligned}
D \tilde{F}\left(\mathbf{x}_{0}, \mathbf{y}_{0}\right)(\mathbf{u})(\mathbf{v}) & =\operatorname{Graph}\left(D \tilde{F}\left(\mathbf{x}_{0}, \mathbf{y}_{0}\right)\right)(\mathbf{u}, \mathbf{v}) \\
& =\tilde{T}\left(\operatorname{Graph}(\tilde{F}) ;\left(\mathbf{x}_{0}, \mathbf{y}_{0}\right)\right)(\mathbf{u}, \mathbf{v}) \\
& \left.=\sup \{\alpha \in] 0,1]:(\mathbf{u}, \mathbf{v}) \in T\left([\operatorname{Graph}(\tilde{F})]_{\alpha} ;\left(\mathbf{x}_{0}, \mathbf{y}_{0}\right)\right)\right\} \\
& \left.=\sup \{\alpha \in] 0,1]:(\mathbf{u}, \mathbf{v}) \in T\left(\operatorname{Graph}\left(F_{\alpha}\right) ;\left(\mathbf{x}_{0}, \mathbf{y}_{0}\right)\right)\right\} \\
& \left.=\sup \{\alpha \in] 0,1]:(\mathbf{u}, \mathbf{v}) \in \operatorname{Graph}\left(D F_{\alpha}\left(\mathbf{x}_{0}, \mathbf{y}_{0}\right)\right)\right\}
\end{aligned}
$$




$$
\begin{aligned}
& \left.=\sup \{\alpha \in] 0,1]: \mathbf{v} \in D F_{\alpha}\left(\mathbf{x}_{0}, \mathbf{y}_{0}\right)(\mathbf{u})\right\} \\
& =M\left(\left\{D F_{\alpha}\left(\mathbf{x}_{0}, \mathbf{y}_{0}\right)(\mathbf{u})\right\}_{\alpha \in] 0,1]}\right)(\mathbf{v}) .
\end{aligned}
$$

The following Proposition 4.4 is a fuzzified one of Proposition 1.10. It can be derived from Proposition 4.1.

Proposition 4.4 Let $\tilde{F}: \mathbb{R}^{n} \rightarrow \mathcal{F}\left(\mathbb{R}^{m}\right)$, and let $\left(\mathbf{x}_{0}, \mathbf{y}_{0}\right) \in[\operatorname{Graph}(\tilde{F})]_{1}$. Then the following statements hold.

(i) $D \tilde{F}\left(\mathbf{x}_{0}, \mathbf{y}_{0}\right)$ is closed-valued.

(ii) If $\operatorname{Graph}(\tilde{F}) \in \mathcal{K} \mathcal{F}\left(\mathbb{R}^{n} \times \mathbb{R}^{m}\right)$, then $D \tilde{F}\left(\mathbf{x}_{0}, \mathbf{y}_{0}\right)$ is closed convex-valued.

\section{Conclusions}

In this article, we proposed definitions of limits of sequences of fuzzy sets, and limits and derivatives of fuzzy set-valued mappings based on level sets of fuzzy sets, and investigated their properties. They are fuzzified ones of them for crisp ones.

Derived results are very general in the sense that they deal with all fuzzy sets, especially fuzzy sets which are not support bounded.

Consider some fuzzy mathematical model whose optimal value/solution output is a fuzzy set for an input parameter. The concepts of limits of sequences of fuzzy sets, limits and derivatives of fuzzy set-valued mappings are necessary and important for stability theory and sensitivity analysis for such fuzzy mathematical models. Then derived results can be expected to be useful for them.

Competing interests

The authors declare that they have no competing interests.

Authors' contributions

MK derived all results communicating with HK. All authors read and approved the final manuscript.

\section{Author details}

${ }^{1}$ Graduate School of Science and Technology, Hirosaki University, 3, Bunkyo, Hirosaki, Aomori 036-8561, Japan. ${ }^{2}$ Faculty of Business Administration and Information Science, Kanazawa Gakuin University, 10, Sue, Kanazawa, Ishikawa 920-1392, Japan.

Received: 19 June 2013 Accepted: 6 November 2013 Published: 02 Dec 2013

\section{References}

1. Aubin, JP, Frankowska, H: Set-Valued Analysis. Birkhäuser, Basel (1990)

2. Maeda, T: Multiobjective Decision Making Theory and Economic Analysis. Makino-Syoten, Japan (1996) (in Japanese)

3. Rockafellar, RT, Wets, RJB: Variational Analysis. Springer, New York (1998)

4. Tanino, T: Theory and applications of set-valued mappings, Part 1: fundamental properties of set-valued mappings. J. Jpn. Soc. Fuzzy Syst. 13,11-19 (2001) (in Japanese)

5. Tanino, T: Theory and applications of set-valued mappings, Part 2: derivatives of set-valued mappings and applications to optimization. J. Jpn. Soc. Fuzzy Syst. 13, 146-154 (2001) (in Japanese)

6. Tanino, T: Theory and applications of set-valued mappings, Part 3: applications of set-valued mappings to dynamical systems, game theory and so on. J. Jpn. Soc. Fuzzy Syst. 13, 234-242 (2001) (in Japanese)

7. Diamond, P, Kloeden, P: Metric Spaces of Fuzzy Sets: Theory and Applications. World Scientific, Singapore (1994)

8. Kurano, M, Yasuda, M, Nakagami, J, Yoshida, Y: Ordering of convex fuzzy sets - a brief survey and new results. J. Oper. Res. Soc. Jpn. 43, 138-148 (2000)

9. Yoshida, Y, Yasuda, M, Nakagami, J, Kurano, M: A limit theorem in dynamic fuzzy systems with a monotone property. Fuzzy Sets Syst. 94, 109-119 (1998)

10. Furukawa, N: Convexity and local Lipschitz continuity of fuzzy-valued mappings. Fuzzy Sets Syst. 93, 113-119 (1998)

11. Furukawa, N: Mathematics of Fuzzy Optimization. Morikita-Syuppan, Japan (1999) (in Japanese)

12. Bazaraa, MS, Goode, JJ, Nashed, MZ: On the cones of tangents with applications to mathematical programming. J. Optim. Theory Appl. 13, 11-19 (1974)

13. Dubois, D, Ostasiewicz, W, Prade, H: Fuzzy sets: history and basic notions. In: Dubois, D, Prade, H (eds.) Fundamentals of Fuzzy Sets, pp. 21-124. Kluwer Academic, Boston (2000)

14. Kon, M: On degree of non-convexity of fuzzy sets. Sci. Math. Jpn. 76, 417-425 (in press) 
10.1186/1687-1812-2013-327

Cite this article as: Kon and Kuwano: On sequences of fuzzy sets and fuzzy set-valued mappings. Fixed Point Theory and Applications 2013, 2013:327

Submit your manuscript to a SpringerOpen ${ }^{\circ}$ journal and benefit from:

- Convenient online submission

- Rigorous peer review

- Immediate publication on acceptance

Open access: articles freely available online

- High visibility within the field

- Retaining the copyright to your article

Submit your next manuscript at $\gg$ springeropen.com 